\title{
INTERACTIONS OF ANISOTROPIC INCLUSIONS ON A FLUID MEMBRANE*
}

\author{
JAMES A. KWIECINSKI ${ }^{\dagger}$, ALAIN GORIELY ${ }^{\ddagger}$, AND S. JON CHAPMAN ${ }^{\ddagger}$
}

Abstract. Biological cells and membranes need to be properly shaped to fulfill many fundamental functions. This shaping is often aided by the aggregation of membrane-bound proteins that both sense the membrane curvature and shape it. Therefore, these protein inclusions interact with each other through the deformation of the membrane that they influence and a key question is to understand the law that governs their interaction. Whereas the theoretical case of isotropic proteins is well understood, an important feature of many such proteins is their anisotropic interaction with the membrane. Here, we derive an interaction law for rigid circular membrane inclusions which impose an anisotropic contact angle on the surrounding membrane. We include the effects of both membrane bending and tension. Using asymptotic analysis, we identify two distinguished limits corresponding to weak anisotropy/weak tension and strong anisotropy/strong tension respectively. The resulting laws exhibit a bi-stability in the equilibrium separation of inclusions. Inclusions with very weak anisotropy equilibrate with large separation, those with very strong anisotropy equilibrate with small separation, while there is a range of anisotropies for which both equilibria are stable. Our results provide a theoretical mechanism for the global aggregation of inclusions seen both in experiments and simulations.

Key words. Membrane-mediated interaction, lipid bilayer, asymptotic analysis

AMS subject classifications. 74G10,

1. Introduction. The shaping of lipid membranes in cells is at the core of many biological processes, from the production and absorption of vesicles, which transport wastes and nutrients, to cell motion $[24,36]$ in both eukaryotic cells, and prokaryotic cells [7]. A key component in membrane-shaping is the attachment of particular families of proteins such as the BAR (Bin/Amphiphysin/Rvs) [27] and ENTH (Epsin N-Terminal Homology) [5] families which induce curvature changes by means of the insertion of an amphipathic helix group into the monolayer of the lipid bilayer [37, 38].

Recent work has found that proteins of a particular mechanical stiffness will be recruited from the surrounding fluid to form patterns of high and low concentrations on simplified membrane geometries in order to reach a final equilibrium shape [22]. However, experimental and computational work has suggested that long-range interactions between proteins which are already attached to the membrane lead to aggregation and that this phenomenon plays a significant role in membrane shaping $[32,34,35,33,39,31,4]$. The nature of interactions between proteins varies from the direct and short range, where van der Waals forces and hydrogen bonds dominate, to more indirect global interactions which are mediated by the underlying membrane shape and mechanical properties [20]. Membrane-mediated interactions can be further split into forces arising from Casimir-like thermal effects, from perturbations in the degrees of freedom of the membrane such as thickness, surface tension, and curvature. The present work considers these latter two effects.

\footnotetext{
*Submitted to the editors [INSERT DATE HERE].
}

Funding: J.A.K. would like to thank the Japan Society for the Promotion of Science for financial support in making this work possible. The support for A.G. by the Engineering and Physical Sciences Research Council of Great Britain under research grant EP/R020205/1 is gratefully acknowledged.

${ }^{\dagger}$ Mathematics, Mechanics, and Materials Unit, Okinawa Institute of Science and Technology, Okinawa, 904-0495 (james.kwiecinski@oist.jp).

${ }^{\ddagger}$ Mathematical Institute, University of Oxford, Andrew Wiles Building, Radcliffe Observatory Quarter, Woodstock Road, Oxford, OX2 6GG, United Kingdom (chapman@maths.ox.ac.uk, Corresponding author: goriely@maths.ox.ac.uk) 
There has been substantial theoretical work describing the global interactions between inclusions embedded in a fluid membrane. In particular, it was found that perfectly circular isotropic inclusions which impose a constant opening angle will repel due to effects from membrane bending with an interaction energy for the separation distance $R$ going to infinity of the form $[17,13]$

$$
\mathcal{E}=\frac{a_{4}}{R^{4}},
$$

where $a_{4}>0$. The above equality and the following forms of the energy should be understood in their asymptotic sense, the details of which will be given in the next sections. Further work has since extended the effects of the curvature imposed by these isotropic inclusions to include spontaneous membrane mean curvature [6,2], lateral membrane tension [40], and slightly non-circular but symmetrical geometries [12] which exhibit attraction depending on the inverse fourth power of the separation and on the inclusion orientation. Higher order expansions of these curvature interactions have been calculated [14], and effective field theories developed [41, 42, 21].

Thermal Casimir-like forces have been suggested as a mechanism for proteins to aggregate, given that attraction does occur for isotropic inclusions $[28,8]$ and rodlike geometries $[16,15]$ with an energy depending on the inverse fourth power of separation, with similar extensions to include membrane tension [23] and mean field theories [9]. However, it is not clear whether this attraction is stronger than the curvature-mediated repulsion [30] that has been theoretically found. It was recently posited that the disagreement between the aggregation observed in experiment and large-scale simulation and the repulsion predicted in theory could be explained by means of inclusions having unique mechanical signatures when they are embedded in the membrane [1].

The study of inclusions which interact anisotropically with the surrounding membrane could provide an explanation as to why and how aggregation occurs. Previous work has shown that such anisotropic inclusions will interact and aggregate [10, 11] due to similar Casimir thermal effects whilst an interaction energy between inclusions proportional to the inverse separation squared was obtained when up-down symmetry was broken [26]. Another approach was taken in recent work [25] where the interaction between rod-shaped inclusions is considered. Both [26] and [25] obtain an interaction energy, for large $R$, of the form

$$
\mathcal{E}=-\frac{\tilde{a}_{2}}{R^{2}},
$$

where $\tilde{a}_{2}$ is a function of the inclusions' orientations. However, this function is substantially different in the two approaches. In [26], $\tilde{a}_{2}$ vanishes in the limit of zero temperature and no attractive effects remain. In [25], $\tilde{a}_{2}$ does not vanish at zero temperature but the theory predicts that inclusions will continue to attract without bound, suggesting that there is no stable equilibrium.

In contrast to [25], in which inclusions are modeled as point singularities regularized using a cut-off in Fourier space, the present work uses matched-asymptotic techniques to study the interaction between small but finite anisotropic inclusions on a fluid membrane.

We find that there are two distinguished limits. When both the anisotropy and tension are weak, we find an interaction energy of the form

$$
\mathcal{E}=-\frac{a_{2}}{R^{2}}+\frac{a_{4}}{R^{4}}-a_{0} \log R,
$$




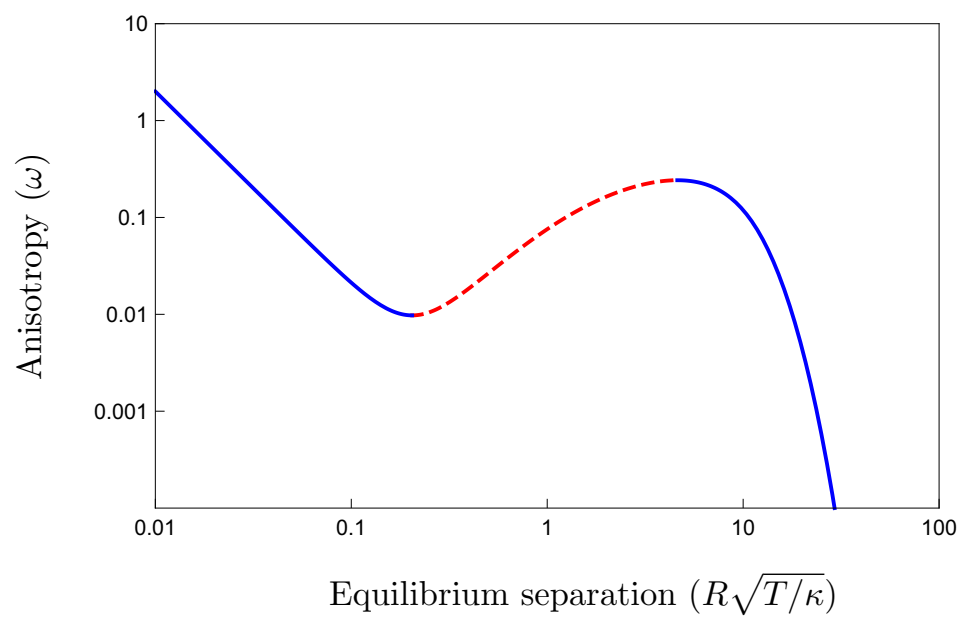

FIG. 1. Dimensionless equilibrium separation $R \sqrt{T / \kappa}$ as a function of anisotropy $\omega$ (defined in §3.1), shown for $\sqrt{\kappa / T r_{0}^{2}}=100$, where $\kappa$ is the membrane bending stiffness, $T$ is the tension, and $r_{0}$ is the radius of the inclusions. The dashed red line corresponds to an unstable equilibrium position, the solid blue lines are stable equilibrium positions.

where $a_{2}$ is a function of the orientation angles of the inclusions with respect to a fixed axis, and $a_{0}$ is proportional to the membrane tension. We show that to leadingorder anisotropic inclusions interact like quadrupoles: their long axes tend to align with each other in an end-to-end configuration. The interaction energy (1.3) gives rise to a finite and non-zero stable equilibrium provided $a_{2}$ is sufficiently large (i.e. the anisotropy is sufficiently strong).

When both the anisotropy and membrane tension are strong, we find an interaction law of the form

$$
\mathcal{E}=\hat{a}_{0} K_{0}(R)+\hat{a}_{2} K_{2}(R)+\hat{a}_{4}\left(K_{4}(R)-\frac{48}{R^{4}}\right),
$$

where $\hat{a}_{0}, \hat{a}_{2}$ and $\hat{a}_{4}$ depend on the orientation angles of the inclusions and the anisotropy, $R$ is a scaled separation distance, and the $K_{i}$ are modified Bessel functions of the second kind. We find that for sufficiently small anisotropy the interaction energy (1.4) also gives rise to a finite and non-zero stable equilibrium. Since strong tension is equivalent to large separation (see the definition of $\mu$ following (2.2)), combining these two results gives an $S$-shaped equilibrium separation as a function of anisotropy, as shown in Fig. 1. This result provides a universal mechanism to explain the global aggregation of membrane inclusions observed in molecular dynamics simulations and in vitro experiments.

\section{Preliminaries.}

2.1. Shape equation. We begin with the Helfrich Hamiltonian which describes the deformation energy of an infinitesimally thin fluid membrane. For such a membrane with mean curvature $H$, intrinsic mean curvature $H_{0}$, and Gaussian curvature $K_{G}$, the energy $E$ of deformation is given by [18]:

$$
E=\int_{\Omega} \frac{\kappa}{2}\left(H-H_{0}\right)^{2}+\bar{\kappa} K_{G}+T \mathrm{~d} A,
$$


where $\kappa$ is the bending stiffness, $\bar{\kappa}$ is the saddle-splay modulus, which is a measure of the membrane's resistance to changes in Gaussian curvature, $T$ is the tension, assumed constant throughout the membrane, and the integral is taken over the entire surface of the lipid bilayer $\Omega$.

We nondimensionalize position with an arbitrary length $L$ (we will later take $L$ to be a typical separation between inclusions), and consider small deformations of an infinite and initially flat membrane in the Monge parametrization. Thus $H_{0}=0$ and the distortions of the membrane are described by the position vector $\boldsymbol{r}=(x, y, \xi h(x, y))$ with $(x, y) \in \Omega$, where $\xi \ll 1$ is a small parameter, and the height function $h$ has been nondimensionalized with $L$. This parametrization gives the mean curvature and the infinitesimal area element as

$$
H=\xi \nabla^{2} h+O\left(\xi^{2}\right), \quad \mathrm{d} A=\left(1+\xi^{2}|\nabla h|^{2} / 2+O\left(\xi^{4}\right)\right) \mathrm{d} x \mathrm{~d} y,
$$

respectively. We assume small slopes so that the quadratic order provides a good approximation of the curvature. Larger slopes may lead to qualitatively different behaviors as suggested by simulations in [29]. Note also that we do not need to consider the Gaussian curvature term because the corresponding energy contribution is constant if the topology of the surface is fixed [3], which we assume, and because the cone angle set by the inclusion is also fixed (in effect, the inclusions are holes, but their geodesic curvature is fixed by the cone angle).

Nondimensionalizing $E$ by writing $E=T A_{0}+\kappa \xi^{2} E^{\prime}$, where $A_{0}$ is the area of the undeformed membrane, and immediately dropping the prime (2.1) becomes, to leading order in $\xi$,

$$
E[h]=\frac{1}{2} \int_{\Omega}\left(\nabla^{2} h\right)^{2}+\mu^{-2}|\nabla h|^{2} \mathrm{~d} x \mathrm{~d} y,
$$

where $\mu=\sqrt{\kappa / T L^{2}}$. By first variation in $h(x, y)$, we find the associated shape equation:

$$
\nabla^{4} h-\mu^{-2} \nabla^{2} h=0 .
$$

2.2. Inclusion boundary conditions. To determine the boundary conditions on (2.3), we now consider a perfectly rigid inclusion embedded in the membrane. For ease of exposition we suppose that the inclusion is flat and described by $\boldsymbol{r}=(x, y, 0)$ with $(x, y) \in D_{0}$. We suppose that, when embedded in the membrane, the inclusion is free to adopt its natural height and orientation, so that it is (vertical) force and (horizontal) moment free. Its position in the membrane is therefore given by

$$
\boldsymbol{r}=\left(x, y, \xi \gamma_{i}+\xi \beta_{i 1}\left(x-x_{i}\right)+\xi \beta_{i 2}\left(y-y_{i}\right)\right)+O\left(\xi^{2}\right),
$$

for $\left(x-x_{i}, y-y_{i}\right) \in D_{0}$, where $\left(x_{i}, y_{i}, \xi \gamma_{i}\right)$ is the position of the center of the inclusion, and $-\xi \beta_{i 1}$ and $\xi \beta_{i 2}$ are the rotations about the $y$ and $x$ axes respectively (to leading order in $\xi$ the order of the rotations does not matter).

We demand that the membrane joins continuously with the inclusion, so that, to leading order in $\xi$,

$$
h=\gamma_{i}+\beta_{i 1}\left(x-x_{i}\right)+\beta_{i 2}\left(y-y_{i}\right) \quad \text { for }(x, y) \in \partial D_{i},
$$

where $D_{i}$ is the projection of the inclusion, so that $(x, y) \in D_{i}$ if and only if $\left(x-x_{i}, y-y_{i}\right) \in D_{0}$. 


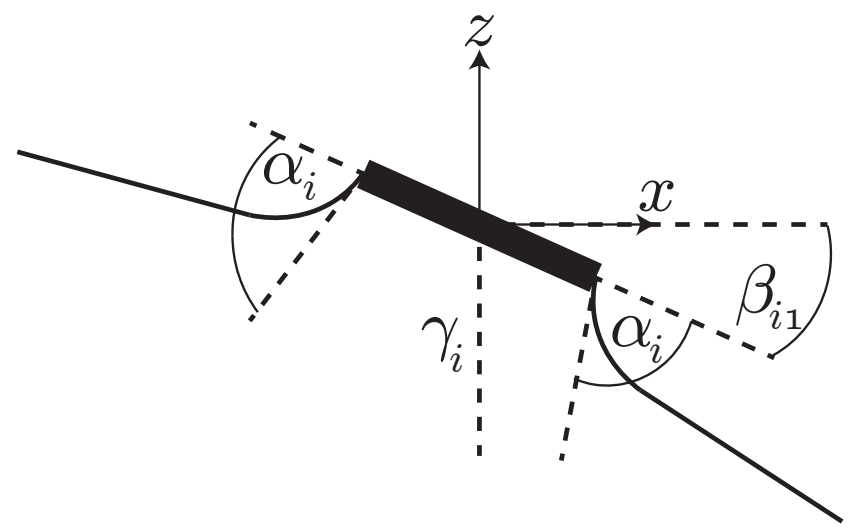

FIG. 2. A schematic diagram of the boundary conditions (i.e. (2.5) and (2.6)) at the membrane inclusion (sketched as the thick black rectangle) in the $x-z$ axis. The inclusion can translate along the $z$-axis, $\gamma_{i}$, and rotate around the $y$-axis, $\beta_{i 1}$, and the $x$-axis, $\beta_{i 2}$ (not shown). The inclusion imposes a contact angle $\alpha_{i}$ with the membrane (denoted by the solid black line). There are also equilibrium conditions on the transverse forces and torques at the boundary (see text).

We now consider the normal derivative of the surrounding membrane with respect to the inclusion boundary in the $x-y$ plane. We suppose that the inclusion enforces a contact angle (equivalent to considering an opening angle, see Fig. 2) of $\alpha_{i}(x, y)$. To find the boundary condition, we set the normal derivative $\partial / \partial \hat{\boldsymbol{n}}_{i}$ of (2.5) with respect to the inclusion boundary to obtain:

$$
\left.\frac{\partial h}{\partial \hat{\boldsymbol{n}}_{i}}\right|_{\partial D_{i}}=\left(\alpha_{i}(x, y)+\beta_{i 1} \frac{\partial x}{\partial \hat{\boldsymbol{n}}_{i}}+\beta_{i 2} \frac{\partial y}{\partial \hat{\boldsymbol{n}}_{i}}\right)_{\partial D_{i}} .
$$

The remaining boundary conditions are given by the balance of vertical force and horizontal torques on the inclusion. These are derived by considering the boundary terms which arise when considering variations of (2.2) with respect to $h$ : namely

$$
0=\oint_{\partial \Omega}\left(\nabla^{2} h \frac{\partial \delta h}{\partial \hat{\boldsymbol{n}}}-\delta h \frac{\partial}{\partial \hat{\boldsymbol{n}}}\left(\nabla^{2} h\right)+\mu^{-2} \delta h \frac{\partial h}{\partial \hat{\boldsymbol{n}}}\right) \cdot \mathrm{d} \boldsymbol{s},
$$

where

$$
\begin{aligned}
\delta h & =\delta \gamma_{i}+\delta \beta_{i 1}\left(x-x_{i}\right)+\delta \beta_{i 2}\left(y-y_{i}\right), \\
\frac{\partial \delta h}{\partial \hat{\boldsymbol{n}}_{i}} & =\delta \beta_{i 1} \frac{\partial x}{\partial \hat{\boldsymbol{n}}_{i}}+\delta \beta_{i 2} \frac{\partial y}{\partial \hat{\boldsymbol{n}}_{i}} .
\end{aligned}
$$

Since $\gamma_{i}, \beta_{i 1}$, and $\beta_{i 2}$ are free parameters, we find the corresponding natural boundary conditions

$$
\begin{aligned}
& 0=\oint_{\partial D_{i}} \frac{\partial}{\partial \hat{\boldsymbol{n}}}\left(\nabla^{2} h-\mu^{-2} h\right) \cdot \mathrm{d} \boldsymbol{s}, \\
& 0=\oint_{\partial D_{i}}\left[\nabla^{2} h \frac{\partial x}{\partial \hat{\boldsymbol{n}}}-\left(x-x_{i}\right) \frac{\partial}{\partial \hat{\boldsymbol{n}}}\left(\nabla^{2} h-\mu^{-2} h\right)\right] \cdot \mathrm{d} \boldsymbol{s}, \\
& 0=\oint_{\partial D_{i}}\left[\nabla^{2} h \frac{\partial y}{\partial \hat{\boldsymbol{n}}}-\left(y-y_{i}\right) \frac{\partial}{\partial \hat{\boldsymbol{n}}}\left(\nabla^{2} h-\mu^{-2} h\right)\right] \cdot \mathrm{d} \boldsymbol{s},
\end{aligned}
$$




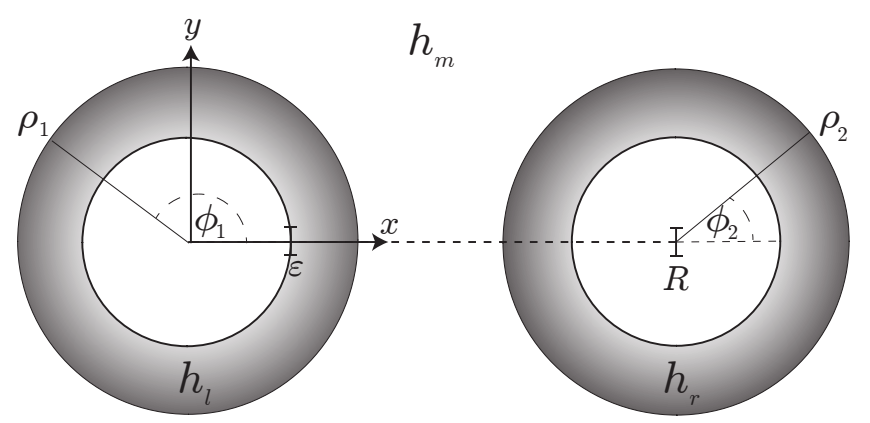

FIG. 3. Two identical inclusions of radius $\varepsilon$ lie on an approximately flat membrane with possibly different contact angles. The inclusions have circular boundaries with centers separated by a distance $R=O(1)$. Three asymptotic regions are illustrated: inner regions near each inclusion parametrized by local radial-angle coordinates $\left(\rho_{i}, \phi_{i}\right)$ (grey region), and the outer membrane region with coordinates $(x, y)$ (white region).

which can be interpreted as a balance of forces in the $z$-direction and a balance of moments around the $y$ and $x$ axes, respectively.

\section{Contact angle anisotropy.}

3.1. Set-up of problem. We now study the interactions between perfectly circular and rigid inclusions which break symmetry in the contact angle that they impose on the surrounding membrane. As shown in Fig 3, we consider two inclusions, denoted by subscripts 1 and 2 , of radius $\varepsilon=r_{0} / L$ with $\varepsilon \ll 1$ whose centers are $R=O(1)$ apart (we take $\left(x_{1}, y_{1}\right)=(0,0)$, and $\left(x_{2}, y_{2}\right)=(R, 0)$ without loss of generality). We parametrize the shape of the membrane in the vicinity of each inclusion by the inner variables given by the local polar radial and angle variables, $\rho_{i} \in[1, \infty), \phi_{i} \in[0,2 \pi)$, measured from the positive $x$-axis. These variables are related to $x$ and $y$ by

$$
\begin{aligned}
x-x_{i} & =\varepsilon \rho_{i} \cos \phi_{i}, \\
y-y_{i} & =\varepsilon \rho_{i} \sin \phi_{i} .
\end{aligned}
$$

We assume that the inclusion enforces an opening angle $\alpha_{i}$ which is not constant but depends on position around the boundary, and thus also on the orientation of the inclusion which we quantify by an angle $\psi_{i}$ measured from the positive $x$-axis:

$$
\alpha_{i}\left(\phi_{i}\right)=\varepsilon^{-1} \alpha_{i}^{*}\left(\phi_{i}-\psi_{i}\right),
$$

for some given non-constant $2 \pi$-periodic function $\alpha_{i}^{*}$. The factor of $\varepsilon^{-1}$ here is included for convenience so that the displacement of the membrane generated by the inclusion is $O(1)$; it is equivalent to a rescaling of $h$. Since $\alpha_{i}^{*}$ is $2 \pi$-periodic we may expand it in a Fourier series as

$$
\alpha_{i}^{*}(\phi)=\alpha_{i}^{(0)}+\alpha_{i}^{(1)} \cos (\phi)+\alpha_{i}^{(2)} \cos (2 \phi)+\cdots .
$$

We will find that the interaction energy does not depend on $\alpha_{i}^{(1)}$ (which only affects the tilts of the inclusion $\beta_{i 1}, \beta_{i 2}$ ). However, the interaction energy does depend strongly on both the characteristic lengthscale $\mu$ over which tension and bending effects are comparable, and the anisotropy $\omega_{i}=\alpha_{i}^{(2)} / \alpha_{i}^{(0)}$. There are two distinguished limits of interest: the case of weak anisotropy/weak tension, and the case of strong anisotropy/strong tension. 
3.2. Weak anisotropy/weak tension. For weak tension, we rescale the problem by setting $\mu=\lambda / \varepsilon$, with $\lambda=O(1)$, and consider weak anisotropy, $\omega_{i}=O\left(\varepsilon^{2}\right)$, by setting

$$
\alpha_{i}(\phi)=\alpha_{i}^{(0)}+\varepsilon \alpha_{i}^{(1)} \cos (\phi)+\varepsilon^{2} \alpha_{i}^{(2)} \cos (2 \phi),
$$

where $\alpha_{i}^{(0)}, \alpha_{i}^{(1)}$ and $\alpha_{i}^{(2)}$ are $O(1)$ constants. The scaling chosen is the distinguished limit in which $\alpha_{i}^{(0)}$, and $\alpha_{i}^{(2)}$ both affect the interaction energy to leading order. By analogy with classic electromagnetism [19], we refer to these constants as the monopole, dipole, and quadrupole contact angles respectively.

For the polar parametrization defined in (3.1) and (3.2), we evaluate (2.5) and (2.6) at the inclusion radius $\left(\rho_{i}=1\right)$ to obtain

$$
\begin{aligned}
\left.h\right|_{\rho_{i}=1}= & \gamma_{i}+\varepsilon \beta_{i 1} \cos \phi_{i}+\varepsilon \beta_{i 2} \sin \phi_{i}, \\
\left.\frac{\partial h}{\partial \rho_{i}}\right|_{\rho_{i}=1}=\alpha_{i}^{(0)} & +\varepsilon \alpha_{i}^{(1)} \cos \left(\phi_{i}-\psi_{i}\right)+\varepsilon^{2} \alpha_{i}^{(2)} \cos \left(2 \phi_{i}-2 \psi_{i}\right) \\
& \quad+\varepsilon \beta_{i 1} \cos \phi_{i}+\varepsilon \beta_{i 2} \sin \phi_{i},
\end{aligned}
$$

where the normal derivative in (3.7) has reduced to the differential operator $-\varepsilon^{-1} \partial / \partial \rho_{i}$.

The integrals (2.10)-(2.12) provide a further three boundary conditions, and for a circular inclusion reduce to

$$
\begin{aligned}
0 & =\int_{0}^{2 \pi}\left[\frac{\partial}{\partial \rho_{i}}\left(\nabla_{\ell}^{2} h-\varepsilon^{4} \lambda^{-2} h\right)\right]_{\rho_{i}=1} \mathrm{~d} \phi_{i}, \\
0 & =\int_{0}^{2 \pi} \cos \phi_{i}\left[\frac{\partial}{\partial \rho_{i}}\left(\nabla_{\ell}^{2} h-\varepsilon^{4} \lambda^{-2} h\right)-\nabla_{\ell}^{2} h\right]_{\rho_{i}=1} \mathrm{~d} \phi_{i}, \\
0 & =\int_{0}^{2 \pi} \sin \phi_{i}\left[\frac{\partial}{\partial \rho_{i}}\left(\nabla_{\ell}^{2} h-\varepsilon^{4} \lambda^{-2} h\right)-\nabla_{\ell}^{2} h\right]_{\rho_{i}=1} \mathrm{~d} \phi_{i},
\end{aligned}
$$

where $\nabla_{\ell} \equiv \varepsilon \nabla$ is the gradient operator with respect to the inner variables.

3.2.1. Method to derive the interaction energy. There are four regions of interest in which we solve (2.3) for the height function $h$ : the two inner regions close to the inclusions, in which we will denote the solution by $h_{l}$ and $h_{r}$ respectively; one outer region on the scale of the separation between the inclusions, in which we will denote the solution by $h_{m}$ (see Fig. 3); and one outer-outer region in which the tension becomes important, with solution $h_{o}$ (see Fig. 4). In each region we expand the membrane shape as a perturbation series in $\varepsilon$. In the inner regions we use the local polar variables $\left(\rho_{i}, \phi_{i}\right)$ and impose the boundary conditions (3.6)-(3.10). In the outer region we use the coordinates $(x, y)$ and the inclusions appear as point singularities. In the outer-outer region we use the coordinates $\left(x^{\prime}, y^{\prime}\right)=(\varepsilon / \lambda)(x, y)$, the inclusions appear as a single singularity, and we impose the far-field condition. By matching the inner, outer, and outer-outer asymptotic expansions we determine the membrane shape, from which we explicitly compute the energy by integrating (2.2).

3.2.2. Leading-order inner solution. We consider the solution near the first inclusion, denoted by $h_{l}$. Expanding as $h_{l}=h_{l}^{(0)}+\varepsilon h_{l}^{(1)}+\cdots$, with corresponding 


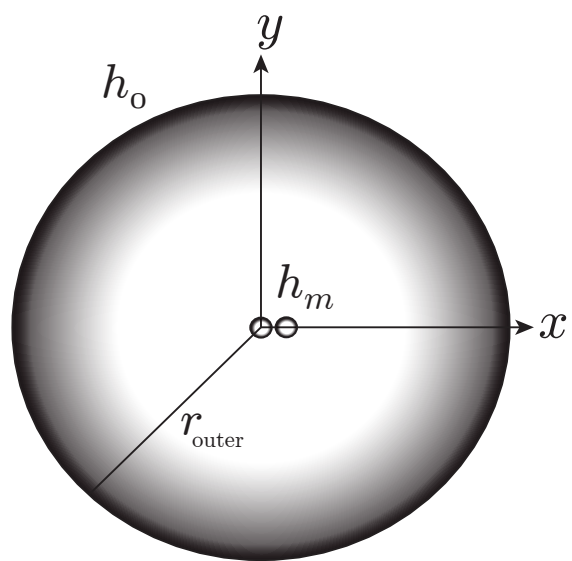

FIG. 4. A schematic diagram depicting the outer and outer-outer solution, $h_{m}$ and $h_{0}$ respectively, far from the inclusions described in Fig. 3. At a large distance from the inclusions, the distance between them is asymptotically small and they appear as one singularity centered at the origin. The outer-outer region corresponds to the scale on which the effects of tension are comparable to those of bending; the membrane shape is parametrized by $r_{\text {outer }}=\sqrt{x^{2}+y^{2}} / \mu$ and determined by (3.15).

expansions for the unknowns $\gamma_{1}, \beta_{11}, \beta_{12}$, we find at leading order that

$$
\nabla_{\ell}^{4} h_{l}^{(0)}=0,\left.\quad h_{l}^{(0)}\right|_{\rho_{1}=1}=\gamma_{1}^{(0)},\left.\quad \frac{\partial h_{l}^{(0)}}{\partial \rho_{1}}\right|_{\rho_{1}=1}=\alpha_{1}^{(0)},
$$

along with (3.8)-(3.10) applied to $h_{l}^{(0)}$, where $\nabla_{\ell}$ is the gradient operator with respect to the inner variables. The solution is

$$
h_{l}^{(0)}=\gamma_{1}^{(0)}+\alpha_{1}^{(0)} \log \rho_{1},
$$

where we preemptively rule out a term proportional to $\rho_{1}^{2}$ as it would grow too large to match with the outer region. A similar solution $h_{r}^{(0)}$ holds in the inner region near the second inclusion.

3.2.3. Leading-order outer solution. Expanding $h_{m}=h_{m}^{(0)}+\varepsilon h_{m}^{(1)}+\cdots$ we find at leading order that

$$
\nabla^{4} h_{m}^{(0)}=0 .
$$

In order to match with the inner solutions the outer solution must have a logarithmic singularity as each inclusion is approached. The solution to (3.12) with these singularities, growing no faster than a logarithm at infinity, is

$$
h_{m}^{(0)}=c_{0}+c_{11} \log \left(x^{2}+y^{2}\right)^{1 / 2}+c_{12} \log \left((x-R)^{2}+y^{2}\right)^{1 / 2},
$$

where $c_{0}, c_{11}$, and $c_{12}$ are constants that will be determined by matching. Writing (3.13) in terms of the inner variables $\left(\rho_{1}, \phi_{1}\right)$ and expanding for small $\varepsilon$ gives

$$
\begin{aligned}
h_{m}^{(0)}=c_{0}+ & c_{11} \log \varepsilon+c_{11} \log \rho+c_{12} \log R \\
& -\frac{\varepsilon c_{12} \rho_{1} \cos \phi_{1}}{R}-\frac{\varepsilon^{2} c_{12} \rho_{1}^{2} \cos 2 \phi_{1}}{2 R^{2}}+O\left(\varepsilon^{3}\right) .
\end{aligned}
$$


Matching (3.14) with (3.11) gives

$$
c_{11}=\alpha_{1}^{(0)}, \quad \gamma_{1}^{(0)}=c_{0}+c_{11} \log \varepsilon+c_{12} \log R .
$$

Similarly matching with the inner region near the second inclusion gives

$$
c_{12}=\alpha_{2}^{(0)}, \quad \gamma_{2}^{(0)}=c_{0}+c_{12} \log \varepsilon+c_{11} \log R .
$$

We observe that the isotropic deformation imposed by both inclusions results in their vertical lift, as evidenced by the dependence of $\gamma_{i}$ on both $\alpha_{1}^{(0)}$ and $\alpha_{2}^{(0)}$, as well as the radius $\varepsilon$ and separation $R$.

3.2.4. Leading-order outer-outer solution. The leading-order outer solution (3.13) has a logarithmic singularity at infinity. We need an outer-outer region, in which tension balances bending, to regularize this singularity and allow the membrane to be flat at infinity. The leading-order outer-outer solution satisfies

$$
\nabla_{o}^{4} h_{o}^{(0)}-\nabla_{o}^{2} h_{o}^{(0)}=0,
$$

where $\nabla_{o}$ is the gradient with respect to the outer-outer variable $\mathbf{x}^{\prime}=\varepsilon \mathbf{x} / \lambda$. The solution which matches with (3.13) is

$$
h_{o}=c_{0}-\left(\alpha_{1}^{(0)}+\alpha_{2}^{(0)}\right)(\gamma-\log (2 \lambda / \varepsilon))-\left(\alpha_{1}^{(0)}+\alpha_{2}^{(0)}\right) K_{0}\left(r_{\text {outer }}\right),
$$

where $r_{\text {outer }}=\left|\mathbf{x}^{\prime}\right|=\varepsilon \sqrt{x^{2}+y^{2}} / \lambda, \gamma \approx 0.5772$ is the Euler-Mascheroni constant, and $K_{0}$ is a modified Bessel function of the second kind.

3.2.5. First-order inner solution. Equating coefficients of $\varepsilon$ in the inner region we find

$$
\nabla_{\ell}^{4} h_{l}^{(1)}=0
$$

with

$$
\begin{aligned}
h_{l}^{(1)} & =\gamma_{1}^{(1)}+\beta_{11}^{(0)} \cos \phi_{1}+\beta_{12}^{(0)} \sin \phi_{1}, \\
\frac{\partial h_{l}^{(1)}}{\partial \rho_{1}} & =\left(\alpha_{1}^{(1)} \cos \psi_{1}+\beta_{11}^{(0)}\right) \cos \phi_{1}+\left(\alpha_{1}^{(1)} \sin \psi_{1}+\beta_{12}^{(0)}\right) \sin \phi_{1},
\end{aligned}
$$

on $\rho_{1}=1$, along with (3.8)-(3.10) applied to $h_{l}^{(1)}$. Matching with (3.14) implies

$$
h_{l}^{(1)} \sim-\frac{\alpha_{2}^{(0)} \rho_{1} \cos \phi_{1}}{R} \quad \text { as } \rho_{1} \rightarrow \infty .
$$

The solution is

$$
h_{l}^{(1)}=\gamma_{1}^{(1)}-\frac{\alpha_{2}^{(0)} \rho_{1} \cos \phi_{1}}{R}-\frac{\alpha_{1}^{(1)} \cos \psi_{1} \cos \phi_{1}}{2 \rho_{1}}-\frac{\alpha_{1}^{(1)} \sin \psi_{1} \sin \phi_{1}}{2 \rho_{1}},
$$

with

$$
\beta_{11}^{(0)}=-\frac{\alpha_{2}^{(0)}}{R}-\frac{\alpha_{1}^{(1)} \cos \psi_{1}}{2}, \quad \beta_{12}^{(0)}=-\frac{\alpha_{1}^{(1)} \sin \psi_{1}}{2} .
$$

We see that the presence of the second inclusion induces a tilt of the first inclusion about the axis perpendicular to the line of centers, while the dipole contact angle $\alpha_{i}^{(1)}$ induces a tilt about an axis perpendicular to the symmetry line of the inclusion. Note also that previous analyses of the isotropic problem $[17,40]$ make the assumption that the membrane height is invariant under the reflection $y \rightarrow-y$. This is no longer true for the case of anisotropic inclusions as can be seen by the presence of the $\sin \psi_{1}$ terms here and later in $h_{l}^{(2)}$. 
3.2.6. First-order outer solution. In order to match with (3.18) and (3.11), the first-order outer solution is simply

$$
h_{m}^{(1)}=\gamma_{1}^{(1)}=\gamma_{2}^{(1)}=0,
$$

where the final equality comes from matching with the outer-outer solution.

3.2.7. Second-order inner solution. The next terms we have to consider are the $O\left(\varepsilon^{2}\right)$ terms in the inner expansion, which satisfy

$$
\nabla_{\ell}^{4} h_{l}^{(2)}=0,
$$

with

$$
\begin{aligned}
\left.h_{l}^{(2)}\right|_{\rho_{1}=1} & =\gamma_{1}^{(2)}+\beta_{11}^{(1)} \cos \phi_{1}+\beta_{12}^{(1)} \sin \phi_{1}, \\
\left.\frac{\partial h_{l}^{(2)}}{\partial \rho_{1}}\right|_{\rho_{1}=1} & =\alpha_{1}^{(2)} \cos \left(2 \phi_{1}-2 \psi_{1}\right)+\beta_{11}^{(1)} \cos \phi_{1}+\beta_{12}^{(1)} \sin \phi_{1},
\end{aligned}
$$

along with (3.8)-(3.10) applied to $h_{l}^{(2)}$. Matching with (3.14) and (3.19) implies

$$
h_{l}^{(2)} \sim-\frac{\alpha_{2}^{(0)} \rho_{1}^{2} \cos 2 \phi_{1}}{2 R^{2}}+O(1) \quad \text { as } \rho_{1} \rightarrow \infty .
$$

The solution is

$$
\begin{aligned}
h_{l}^{(2)}= & \gamma_{1}^{(2)}-\frac{\alpha_{2}^{(0)} \rho_{1}^{2} \cos 2 \phi_{1}}{2 R^{2}}+\left(\frac{\alpha_{2}^{(0)}}{R^{2}}+\frac{\alpha_{1}^{(2)} \cos 2 \psi_{1}}{2}\right) \cos 2 \phi_{1} \\
& +\frac{\alpha_{1}^{(2)} \sin 2 \psi_{1}}{2} \sin 2 \phi_{1}-\left(\frac{\alpha_{2}^{(0)}}{2 R^{2}}+\frac{\alpha_{1}^{(2)} \cos 2 \psi_{1}}{2}\right) \frac{\cos 2 \phi_{1}}{\rho_{1}^{2}} \\
& -\frac{\alpha_{1}^{(2)} \sin 2 \psi_{1}}{2} \frac{\sin 2 \phi_{1}}{\rho_{1}^{2}}
\end{aligned}
$$

with $\beta_{11}^{(1)}=\beta_{12}^{(1)}=0$. We could now proceed to determine the terms in the secondorder outer solution induced by $h_{l}^{(1)}$ and $h_{l}^{(2)}$, but we already have enough information to determine the energy of interaction to leading order in $\varepsilon$.

3.2.8. Interaction energy. Evaluating the energy density

$$
e=\frac{1}{2}\left(\left(\nabla^{2} h\right)^{2}+\mu^{-2}|\nabla h|^{2}\right),
$$

in each asymptotic region we find to leading order in $\varepsilon$,

$$
\begin{aligned}
e_{\text {inner }_{1}} & =\frac{2\left(2 \alpha_{2}^{(0)} \cos \left(2 \phi_{1}\right)+\alpha_{1}^{(2)} R^{2} \cos \left(2\left(\phi_{1}-\psi_{1}\right)\right)\right)^{2}}{R^{4} \rho_{1}^{4}}+\frac{\left(\alpha_{1}^{(0)}\right)^{2}}{2 \rho_{1}^{2} \lambda^{2}}, \\
e_{\text {inner }_{2}} & =\frac{2\left(2 \alpha_{1}^{(0)} \cos \left(2 \phi_{2}\right)+\alpha_{2}^{(2)} R^{2} \cos \left(2\left(\phi_{2}-\psi_{2}\right)\right)\right)^{2}}{R^{4} \rho_{2}^{4}}+\frac{\left(\alpha_{2}^{(0)}\right)^{2}}{2 \rho_{2}^{2} \lambda^{2}}, \\
e_{\text {outer }} & =\varepsilon^{2} \frac{\left(\alpha_{1}^{(0)} R-\left(\alpha_{1}^{(0)}+\alpha_{2}^{(0)}\right) x\right)^{2}+\left(\alpha_{1}^{(0)}+\alpha_{2}^{(0)}\right)^{2} y^{2}}{2 \lambda^{2}\left((R-x)^{2}+y^{2}\right)\left(x^{2}+y^{2}\right)}, \\
e_{\text {outer-outer }} & =\varepsilon^{4} \frac{\left(\alpha_{1}^{(0)}+\alpha_{2}^{(0)}\right)^{2}}{2 \lambda^{4}}\left(K_{0}\left(r_{\text {outer }}\right)^{2}+K_{1}\left(r_{\text {outer }}\right)^{2}\right),
\end{aligned}
$$


where $r_{\text {outer }}=\varepsilon \sqrt{x^{2}+y^{2}} / \lambda$. All regions contribute equally, and there are logarithmic singularities in the outer region at both infinity and at each inclusion. To cope with these singularities we divide the integral of the energy density into four regions: a region of radius $\delta$ around each inclusion, a region of radius $\eta$ centered at the origin excluding these, and the remaining region near infinity, with $\varepsilon \ll \delta \ll 1$ and $1 \ll$ $\eta \ll \lambda / \varepsilon$. In each region the corresponding asymptotic expansion (inner, outer, and outer-outer) is valid. Then, we have

$$
\begin{gathered}
E=\pi \varepsilon^{2}\left[\left(\alpha_{1}^{(2)}\right)^{2}+\left(\alpha_{2}^{(2)}\right)^{2}+\frac{4}{R^{2}}\left(\alpha_{1}^{(2)} \alpha_{2}^{(0)} \cos 2 \psi_{1}+\alpha_{2}^{(2)} \alpha_{1}^{(0)} \cos 2 \psi_{2}\right)\right. \\
\left.+\frac{4}{R^{4}}\left(\left(\alpha_{1}^{(0)}\right)^{2}+\left(\alpha_{2}^{(0)}\right)^{2}\right)\right] \\
+2 \pi \varepsilon^{2} \frac{\left(\alpha_{1}^{(0)}\right)^{2}+\left(\alpha_{2}^{(0)}\right)^{2}}{2 \lambda^{2}} \log (\delta / \varepsilon)+E_{\text {outer }}+E_{\text {outer-outer }} .
\end{gathered}
$$

To evaluate the integral $E_{\text {outer }}$ we write it as

$$
\begin{aligned}
& E_{\text {outer }}= \int_{|\mathbf{x}|>\delta,|\mathbf{x}-(R, 0)|>\delta,|\mathbf{x}|<\eta} \varepsilon^{2} \frac{\left(\alpha_{1}^{(0)} R-\left(\alpha_{1}^{(0)}+\alpha_{2}^{(0)}\right) x\right)^{2}+\left(\alpha_{1}^{(0)}+\alpha_{2}^{(0)}\right)^{2} y^{2}}{2 \lambda^{2}\left((x-R)^{2}+y^{2}\right)\left(x^{2}+y^{2}\right)} \mathrm{d} x \mathrm{~d} y \\
&= \frac{\varepsilon^{2}}{2 \lambda^{2}} \int_{|\mathbf{x}|>\delta,|\mathbf{x}-(R, 0)|>\delta,|\mathbf{x}|<\eta}\left(2 \alpha_{1}^{(0)} \alpha_{2}^{(0)} \frac{(x-R) x+y^{2}}{\left((x-R)^{2}+y^{2}\right)\left(x^{2}+y^{2}\right)}\right. \\
&\left.+\frac{\left(\alpha_{1}^{(0)}\right)^{2}}{x^{2}+y^{2}}+\frac{\left(\alpha_{2}^{(0)}\right)^{2}}{(x-R)^{2}+y^{2}}\right) \mathrm{d} x \mathrm{~d} y \\
&=2 \pi \varepsilon^{2} \frac{\left(\alpha_{1}^{(0)}\right)^{2}+\left(\alpha_{2}^{(0)}\right)^{2}}{2 \lambda^{2}} \log (\eta / \delta) \\
&+\frac{\alpha_{1}^{(0)} \alpha_{2}^{(0)} \varepsilon^{2}}{\lambda^{2}} \int_{|\mathbf{x}|<\eta} \frac{(x-R) x+y^{2}}{\left((x-R)^{2}+y^{2}\right)\left(x^{2}+y^{2}\right)} \mathrm{d} x \mathrm{~d} y+O(\delta) \\
&=2 \pi \varepsilon^{2} \frac{\left(\alpha_{1}^{(0)}\right)^{2}+\left(\alpha_{2}^{(0)}\right)^{2}}{2 \lambda^{2}} \log (\eta / \delta)+\frac{2 \pi \alpha_{1}^{(0)} \alpha_{2}^{(0)} \varepsilon^{2}}{\lambda^{2}} \log (\eta / R)+O(\delta) .
\end{aligned}
$$

The $\log \delta$ terms cancel with those in (3.21) as they should. The coefficient of $\log \eta$ is exactly what is needed to cancel the $\log \eta$ term in the outer-outer region. For that integral we remove the singularity at the origin by writing

$$
\begin{aligned}
& E_{\text {outer-outer }}=2 \pi \varepsilon^{2} \frac{\left(\alpha_{1}^{(0)}+\alpha_{2}^{(0)}\right)^{2}}{2 \lambda^{2}} \int_{\varepsilon \eta / \lambda}^{\infty}\left(K_{0}\left(r_{\text {outer }}\right)^{2}+K_{1}\left(r_{\text {outer }}\right)^{2}\right) r_{\text {outer }} \mathrm{d} r_{\text {outer }} \\
&=2 \pi \varepsilon^{2} \frac{\left(\alpha_{1}^{(0)}+\alpha_{2}^{(0)}\right)^{2}}{2 \lambda^{2}} \int_{\frac{\varepsilon \eta}{\lambda}}^{1}\left(K_{0}\left(r_{\text {outer }}\right)^{2}+K_{1}\left(r_{\text {outer }}\right)^{2}-\frac{1}{r_{\text {outer }}^{2}}\right) r_{\text {outer }} \mathrm{d} r_{\text {outer }} \\
&-2 \pi \varepsilon^{2} \frac{\left(\alpha_{1}^{(0)}+\alpha_{2}^{(0)}\right)^{2}}{2 \lambda^{2}} \log (\varepsilon \eta / \lambda) \\
&+2 \pi \varepsilon^{2} \frac{\left(\alpha_{1}^{(0)}+\alpha_{2}^{(0)}\right)^{2}}{2 \lambda^{2}} \int_{1}^{\infty}\left(K_{0}\left(r_{\text {outer }}\right)^{2}+K_{1}\left(r_{\text {outer }}\right)^{2}\right) r_{\text {outer }} \mathrm{d} r_{\text {outer }} \\
&=- 2 \pi \varepsilon^{2} \frac{\left(\alpha_{1}^{(0)}+\alpha_{2}^{(0)}\right)^{2}}{2 \lambda^{2}} \log (\varepsilon \eta / \lambda)+2 \pi \varepsilon^{2} \frac{\left(\alpha_{1}^{(0)}+\alpha_{2}^{(0)}\right)^{2}}{2 \lambda^{2}}(-\gamma+\log 2)
\end{aligned}
$$


Thus, the total energy is

$$
\begin{aligned}
(3.22) \frac{E}{\pi \varepsilon^{2}}= & \left(\alpha_{1}^{(2)}\right)^{2}+\left(\alpha_{2}^{(2)}\right)^{2}-\frac{\left(\alpha_{1}^{(0)}\right)^{2}+\left(\alpha_{2}^{(0)}\right)^{2}}{\lambda^{2}} \log (\varepsilon)-\frac{2 \alpha_{1}^{(0)} \alpha_{2}^{(0)}}{\lambda^{2}} \log (R) \\
& +\frac{\left(\alpha_{1}^{(0)}+\alpha_{2}^{(0)}\right)^{2}}{\lambda^{2}}(\log (2 \lambda / \varepsilon)-\gamma)+\frac{4}{R^{4}}\left(\left(\alpha_{1}^{(0)}\right)^{2}+\left(\alpha_{2}^{(0)}\right)^{2}\right) \\
& +\frac{4}{R^{2}}\left(\alpha_{1}^{(2)} \alpha_{2}^{(0)} \cos 2 \psi_{1}+\alpha_{2}^{(2)} \alpha_{1}^{(0)} \cos 2 \psi_{2}\right)
\end{aligned}
$$

We discuss (3.22) further in Sections 4 and 5.

3.3. Strong anisotropy/strong tension. The second distinguished limit we consider corresponds to strong anisotropy and strong tension. We consider the case in which the anisotropy $\omega_{i}=O(1)$ and $\mu=O(1)$ as $\varepsilon \rightarrow 0$. In this case the tension appears at leading order in the outer region, and there is no outer-outer region. Since we have seen that the dipole term $\alpha_{i}^{(1)} \cos \phi$ does not affect the interaction energy, we simplify the discussion by taking $\alpha_{i}^{(1)}=0$.

3.3.1. Leading-order inner solution. In the inner region

$$
\nabla_{l}^{4} h_{l}-\varepsilon^{2} \mu^{-2} \nabla_{l}^{2} h_{l}=0 .
$$

Expanding $h_{l}=h_{l}^{(0)}+\varepsilon h_{l}^{(1)}+\cdots$, with corresponding expansions for the unknowns $\gamma_{1}, \beta_{11}, \beta_{12}$, we find at leading order that

$$
\nabla_{l}^{4} h_{l}^{(0)}=0,\left.\quad h_{l}^{(0)}\right|_{\rho_{1}=1}=\gamma_{1}^{(0)},\left.\quad \frac{\partial h_{l}^{(0)}}{\partial \rho_{1}}\right|_{\rho_{1}=1}=\alpha_{1}^{(0)}+\alpha_{1}^{(2)} \cos \left(2 \phi_{1}-2 \psi_{1}\right),
$$

along with (3.8)-(3.10) applied to $h_{l}^{(0)}$. The solution is

$$
\begin{aligned}
h_{l}^{(0)}=\gamma_{1}^{(0)} & +\alpha_{1}^{(0)} \log \rho_{1}+\frac{\alpha_{1}^{(2)} \cos 2 \psi_{1}}{2} \cos 2 \phi_{1}+\frac{\alpha_{1}^{(2)} \sin 2 \psi_{1}}{2} \sin 2 \phi_{1} \\
& -\frac{\alpha_{1}^{(2)} \cos 2 \psi_{1}}{2} \frac{\cos 2 \phi_{1}}{\rho_{1}^{2}}-\frac{\alpha_{1}^{(2)} \sin 2 \psi_{1}}{2} \frac{\sin 2 \phi_{1}}{\rho_{1}^{2}}
\end{aligned}
$$

where we ruled out terms proportional to $\rho_{1}^{2}$ since they would grow too large to match with the outer region.

3.3.2. Leading-order outer solution. Expanding $h_{m}=h_{m}^{(0)}+\varepsilon h_{m}^{(1)}+\cdots$ we find at leading order that

$$
\nabla^{4} h_{m}^{(0)}-\mu^{-2} \nabla^{2} h_{m}^{(0)}=0 .
$$

In order to match with the inner solutions the outer solution must have the correct singularities as each inclusion is approached. The solution to (3.24) with these singularities and decaying at infinity is

$$
\begin{aligned}
h_{m}^{(0)}= & c_{0}+c_{11} K_{0}\left(r_{1} / \mu\right)+c_{12} K_{0}\left(r_{2} / \mu\right) \\
& +c_{21} \cos \left(2 \phi_{1}-2 \psi_{1}\right) f\left(r_{1} / \mu\right)+c_{22} \cos \left(2 \phi_{2}-2 \psi_{2}\right) f\left(r_{2} / \mu\right),
\end{aligned}
$$

where

$r_{1}=\left(x^{2}+y^{2}\right)^{1 / 2}, \quad \phi_{1}=\tan ^{-1} y / x, \quad r_{2}=\left((x-R)^{2}+y^{2}\right)^{1 / 2}, \quad \phi_{2}=\tan ^{-1} y /(x-R)$, 
and

$$
f(r)=\frac{4}{r^{2}}-2 K_{2}(r),
$$

where $K_{2}$ is a modified Bessel function, so that $f \rightarrow 0$ as $r \rightarrow \infty$ and

$$
f \sim 1+\frac{r^{2} \log (r / 2)}{4}+\frac{(4 \gamma-3) r^{2}}{16} \text { as } r \rightarrow 0,
$$

where $\gamma$ is the Euler-Mascheroni constant. Expanding (3.25) in terms of inner variables $\left(\rho_{1}, \phi_{1}\right)$ gives

$$
\begin{aligned}
h_{m}^{(0)}= & c_{0}-c_{11}\left(\gamma+\log \left(\varepsilon \rho_{1} / 2 \mu\right)\right)+c_{12} K_{0}(R / \mu)+\frac{c_{12} K_{1}(R / \mu)}{\mu} \varepsilon \rho_{1} \cos \phi_{1} \\
& +\frac{c_{11}}{4}\left(1-\gamma+\log 2-\log \varepsilon \rho_{1} / \mu\right) \frac{\varepsilon^{2} \rho_{1}^{2}}{\mu^{2}}+\frac{c_{12} K_{0}(R / \mu)}{2 \mu^{2}} \varepsilon^{2} \rho_{1}^{2} \cos ^{2} \phi_{1} \\
& +\frac{c_{12} K_{1}(R / \mu)}{2 R \mu} \varepsilon^{2} \rho_{1}^{2} \cos 2 \phi_{1} \\
& +c_{21} \cos \left(2 \phi_{1}-2 \psi_{1}\right)\left(1+\frac{\varepsilon^{2} \rho_{1}^{2} \log \left(\varepsilon \rho_{1} / 2 \mu\right)}{4 \mu^{2}}+\frac{(4 \gamma-3) \varepsilon^{2} \rho_{1}^{2}}{16 \mu^{2}}+\cdots\right) \\
& +c_{22} \cos \left(2 \psi_{2}\right)\left[f(R / \mu)-\frac{\varepsilon \rho_{1} \cos \left(\phi_{1}\right) f^{\prime}(R / \mu)}{\mu}\right. \\
& \left.\quad+\varepsilon^{2} \rho_{1}^{2}\left(-\frac{2 f(R / \mu) \sin ^{2} \phi_{1}}{R^{2}}+\frac{\sin ^{2} \phi_{1} f^{\prime}(R / \mu)}{2 R \mu}+\frac{\cos ^{2} \phi_{1} f^{\prime \prime}(R / \mu)}{2 \mu^{2}}\right)\right] \\
& +c_{22} \sin \left(2 \psi_{2}\right)\left[-\frac{2 \varepsilon \rho_{1} f(R / \mu) \sin \phi_{1}}{R}\right. \\
& \left.+\varepsilon^{2} \rho_{1}^{2}\left(-\frac{2 \cos \phi_{1} f(R / \mu) \sin \phi_{1}}{R^{2}}+\frac{2 \cos \phi_{1} \sin \phi_{1} f^{\prime}(R / \mu)}{R \mu}\right)\right]+\cdots .
\end{aligned}
$$

Matching (3.26) with (3.23) gives

$$
\begin{gathered}
c_{11}=-\alpha_{1}^{(0)}, \quad c_{21}=\frac{\alpha_{1}^{(2)}}{2}, \\
\gamma_{1}^{(0)}=c_{0}-c_{11}\left(\log \frac{\varepsilon}{2 \mu}+\gamma\right)+c_{12} K_{0}(R / \mu)+c_{22} \cos \left(2 \psi_{2}\right)\left(\frac{4 \mu^{2}}{R^{2}}-2 K_{2}(R / \mu)\right) .
\end{gathered}
$$

Similarly matching with the inner region near the second inclusion gives

$$
\begin{gathered}
c_{12}=-\alpha_{2}^{(0)}, \quad c_{22}=\frac{\alpha_{2}^{(2)}}{2}, \\
\gamma_{2}^{(0)}=c_{0}-c_{12}\left(\log \frac{\varepsilon}{2 \mu}+\gamma\right)+c_{11} K_{0}(R / \mu)+c_{21} \cos \left(2 \psi_{1}\right)\left(\frac{4 \mu^{2}}{R^{2}}-2 K_{2}(R / \mu)\right) .
\end{gathered}
$$

3.3.3. First-order inner solution. Equating coefficients of $\varepsilon$ in the inner region we find

$$
\nabla_{l}^{4} h_{l}^{(1)}=0
$$

with

$$
h_{l}^{(1)}=\gamma_{1}^{(1)}+\beta_{11}^{(0)} \cos \phi_{1}+\beta_{12}^{(0)} \sin \phi_{1}, \quad \frac{\partial h_{l}^{(1)}}{\partial \rho_{1}}=\beta_{11}^{(0)} \cos \phi_{1}+\beta_{12}^{(0)} \sin \phi_{1}
$$


411

with

$$
\begin{aligned}
& h_{l}^{(1)} \sim-\frac{\alpha_{2}^{(0)} K_{1}(R / \mu)}{\mu} \rho_{1} \cos \phi_{1}-\frac{\alpha_{2}^{(2)}}{2} \cos \left(2 \psi_{2}\right) \frac{\rho_{1} \cos \left(\phi_{1}\right) f^{\prime}(R / \mu)}{\mu} \\
& -\frac{\alpha_{2}^{(2)}}{2} \sin \left(2 \psi_{2}\right) \frac{2 \rho_{1} f(R / \mu) \sin \phi_{1}}{R}+\cdots \quad \text { as } \rho_{1} \rightarrow \infty \text {. }
\end{aligned}
$$

The solution is

$$
h_{l}^{(1)}=\gamma_{1}^{(1)}+\beta_{11}^{(0)} \rho_{1} \cos \phi_{1}+\beta_{12}^{(0)} \rho_{1} \sin \phi_{1}
$$

with (3.27) then giving

$$
\beta_{11}^{(0)}=-\frac{\alpha_{2}^{(0)} K_{1}(R / \mu)}{\mu}-\frac{\alpha_{2}^{(2)} \cos \left(2 \psi_{2}\right) f^{\prime}(R / \mu)}{2 \mu}, \quad \beta_{12}^{(0)}=-\frac{\alpha_{2}^{(2)} \sin \left(2 \psi_{2}\right) f(R / \mu)}{R} .
$$

3.3.4. First-order outer solution. In order to match with (3.28), the firstorder outer solution must be

$$
h_{m}^{(1)}=\gamma_{1}^{(1)}=\gamma_{2}^{(1)}
$$

3.3.5. Second-order inner solution. The next terms we have to consider are the $O\left(\varepsilon^{2}\right)$ terms in the inner expansion, which satisfy

$$
\nabla_{l}^{4} h_{l}^{(2)}=\mu^{-2} \nabla_{l}^{2} h_{l}^{(0)}=-\frac{2 \alpha_{1}^{(2)} \cos \left(2 \phi_{1}-2 \psi_{1}\right)}{\mu^{2} \rho^{2}}
$$

$$
h_{l}^{(2)}=\gamma_{1}^{(2)}+\beta_{11}^{(1)} \cos \phi_{1}+\beta_{12}^{(1)} \sin \phi_{1}, \quad \frac{\partial h_{l}^{(2)}}{\partial \rho_{1}}=\beta_{11}^{(1)} \cos \phi_{1}+\beta_{12}^{(1)} \sin \phi_{1},
$$

on $\rho_{1}=1$, along with (3.8)-(3.10) applied to $h_{l}^{(2)}$. To match with (3.26) we require

$h_{\ell}^{(2)} \sim-\frac{\alpha_{1}^{(0)}}{4}\left(1-\gamma-\log \left(\varepsilon \rho_{1} / 2 \mu\right)\right) \frac{\rho_{1}^{2}}{\mu^{2}}-\frac{\alpha_{2}^{(0)} K_{0}(R / \mu)}{4 \mu^{2}} \rho_{1}^{2}-\alpha_{2}^{(0)} \frac{K_{2}(R / \mu)}{4 \mu^{2}} \rho_{1}^{2} \cos 2 \phi_{1}$

$$
+\frac{\alpha_{1}^{(2)}}{8 \mu^{2}} \cos \left(2 \phi_{1}-2 \psi_{1}\right) \rho_{1}^{2}\left(\log \left(\varepsilon \rho_{1} / 2 \mu\right)+\gamma-\frac{3}{4}\right)-\frac{\alpha_{2}^{(2)}}{4 \mu^{2}} \cos \left(2 \psi_{2}\right) \rho_{1}^{2} K_{2}(R / \mu)
$$$$
+\frac{\alpha_{2}^{(2)}}{2} \cos \left(2 \psi_{2}\right) \cos \left(2 \phi_{1}\right) \rho_{1}^{2}\left(\frac{12 \mu^{2}}{R^{4}}-\left(\frac{1}{2 \mu^{2}}+\frac{6}{R^{2}}\right) K_{0}(R / \mu)\right.
$$$$
\left.-\left(\frac{2}{R \mu}+\frac{12 \mu}{R^{3}}\right) K_{1}(R / \mu)\right)
$$$$
+\frac{\alpha_{2}^{(2)}}{2} \sin \left(2 \psi_{2}\right) \sin \left(2 \phi_{1}\right) \rho_{1}^{2}\left(-\frac{12 \mu^{2}}{R^{4}}+\frac{2}{R \mu} K_{1}(R / \mu)+\frac{6}{R^{2}} K_{2}(R / \mu)\right)
$$

as $\rho_{1} \rightarrow \infty$. 
The solution is

$$
\begin{aligned}
& h_{l}^{(2)}=\gamma_{1}^{(2)}+\frac{\alpha_{1}^{(0)} \rho_{1}^{2} \log \rho_{1}}{4 \mu^{2}} \\
& +\frac{\left(\rho_{1}^{2}-1-2 \log \rho_{1}\right)}{4 \mu^{2}}\left(\alpha_{1}^{(0)}(\gamma-1+\log (\varepsilon / 2 \mu))\right. \\
& \left.\quad-\alpha_{2}^{(0)} K_{0}(R / \mu)-\alpha_{2}^{(2)} \cos \left(2 \psi_{2}\right) K_{2}(R / \mu)\right) \\
& -\alpha_{2}^{(0)}\left(\rho_{1}^{2}-2+\frac{1}{\rho_{1}^{2}}\right) \cos \left(2 \phi_{1}\right) \frac{K_{2}(R / \mu)}{4 \mu^{2}} \\
& +\frac{\rho_{1}^{2} \alpha_{1}^{(2)}}{8 \mu^{2}} \cos \left(2 \phi_{1}-2 \psi_{1}\right)\left(\gamma-\frac{3}{4}+\log \left(\varepsilon \rho_{1} / 2 \mu\right)\right) \\
& +\frac{\alpha_{2}^{(2)}}{2} \cos \left(2 \psi_{2}\right)\left(\rho_{1}^{2}-2+\frac{1}{\rho_{1}^{2}}\right) \cos \left(2 \phi_{1}\right)\left(\frac{12 \mu^{2}}{R^{4}}-\left(\frac{1}{2 \mu^{2}}+\frac{6}{R^{2}}\right) K_{0}(R / \mu)\right. \\
& \left.+\frac{\alpha_{2}^{(2)}}{2} \sin \left(2 \psi_{2}\right)\left(\rho_{1}^{2}-2+\frac{1}{\rho_{1}^{2}}\right) \sin \left(2 \phi_{1}\right)\left(-\frac{12 \mu}{R^{3}}\right) K_{1}(R / \mu)\right) \\
& +\frac{\alpha_{1}^{(2)}}{16 \mu^{2}} \cos \left(2 \phi_{1}-2 \psi_{1}\right)\left(\frac{1}{\rho_{1}^{2}}\left(2 \gamma-\frac{1}{2}+2 \log \frac{\varepsilon}{2 \mu}+2-4 \gamma-4 \log \frac{\varepsilon}{2 \mu}\right)-\frac{\alpha_{1}^{(0)}}{4 \mu^{2}} \log \rho_{1},\right.
\end{aligned}
$$

with $\beta_{11}^{(1)}=\beta_{12}^{(1)}=0$.

3.4. Interaction energy. In this parameter regime the easiest way to evaluate the energy is to integrate by parts:

$$
E=\frac{1}{2} \int_{\Omega}\left(\left(\nabla^{2} h\right)^{2}+\mu^{-2}|\nabla h|^{2}\right) \mathrm{d} \mathbf{x}=\frac{1}{2} \int_{\partial \Omega}\left(\nabla^{2} h \frac{\partial h}{\partial n}-h \frac{\partial}{\partial n} \nabla^{2} h+\mu^{-2} h \frac{\partial h}{\partial n}\right) \mathrm{d} s
$$

where, using the shape equation (3.24) and imposing $h \rightarrow 0$ at infinity, the expression reduces to closed contour integrals around each inclusion. The leading-order inner solution produces a non-zero but constant $O\left(\varepsilon^{-2}\right)$ contribution, so we must evaluate up to $O(1)$ to find the dependence of the energy on $R, \psi_{1}$, and $\psi_{2}$. The result is

$$
\begin{aligned}
\frac{E}{\pi}= & \left(\left(\alpha_{1}^{(2)}\right)^{2}+\left(\alpha_{2}^{(2)}\right)^{2}\right)\left(\frac{1}{\varepsilon^{2}}-\frac{\gamma}{2 \mu^{2}}-\frac{\log (\varepsilon / 2 \mu)}{2 \mu^{2}}\right) \\
& -\left(\left(\alpha_{1}^{(0)}\right)^{2}+\left(\alpha_{2}^{(0)}\right)^{2}\right)\left(\frac{\gamma}{\mu^{2}}+\frac{\log (\varepsilon /(2 \mu))}{\mu^{2}}\right)+\frac{2 \alpha_{1}^{(0)} \alpha_{2}^{(0)}}{\mu^{2}} K_{0}(R / \mu) \\
& +\frac{2 \alpha_{1}^{(2)} \alpha_{2}^{(0)}}{\mu^{2}} \cos \left(2 \psi_{1}\right) K_{2}(R / \mu)+\frac{2 \alpha_{1}^{(0)} \alpha_{2}^{(2)}}{\mu^{2}} \cos \left(2 \psi_{2}\right) K_{2}(R / \mu) \\
& +\frac{\alpha_{1}^{(2)} \alpha_{2}^{(2)}}{\mu^{2}} \cos \left(2\left(\psi_{1}+\psi_{2}\right)\right)\left(-\frac{48 \mu^{4}}{R^{4}}+K_{4}(R / \mu)\right) \\
& +\frac{\alpha_{1}^{(2)} \alpha_{2}^{(2)}}{\mu^{2}} \cos \left(2\left(\psi_{1}-\psi_{2}\right)\right) K_{0}(R / \mu) .
\end{aligned}
$$

4. Summary. A similar analysis can be performed with any combination of weak/strong anisotropy, and weak/strong tension. 
We summarize the results of all such analyses here. For clarity we remove the constant terms from the energy.

Weak tension/weak anisotropy. With $\mu=\lambda / \varepsilon, \alpha_{1}^{(2)}=\varepsilon^{2} \bar{\alpha}_{1}^{(2)}, \alpha_{2}^{(2)}=\varepsilon^{2} \bar{\alpha}_{2}^{(2)}$,

$$
\begin{aligned}
\frac{E}{\pi \varepsilon^{2}}= & \frac{4}{R^{2}}\left(\bar{\alpha}_{1}^{(2)} \alpha_{2}^{(0)} \cos 2 \psi_{1}+\bar{\alpha}_{2}^{(2)} \alpha_{1}^{(0)} \cos 2 \psi_{2}\right) \\
& +\frac{4}{R^{4}}\left(\left(\alpha_{1}^{(0)}\right)^{2}+\left(\alpha_{2}^{(0)}\right)^{2}\right)-\frac{2 \alpha_{1}^{(0)} \alpha_{2}^{(0)}}{\lambda^{2}} \log (R)
\end{aligned}
$$

Strong tension/weak anisotropy. With $\alpha_{1}^{(2)}=\varepsilon^{2} \bar{\alpha}_{1}^{(2)}, \alpha_{2}^{(2)}=\varepsilon^{2} \bar{\alpha}_{2}^{(2)}$,

$$
\frac{E}{\pi}=\frac{2 \alpha_{1}^{(0)} \alpha_{2}^{(0)}}{\mu^{2}} K_{0}(R / \mu)
$$

Weak tension/strong anisotropy. With $\mu=\lambda / \varepsilon$,

$$
\frac{E}{\pi}=\frac{4}{R^{2}}\left(\alpha_{1}^{(2)} \alpha_{2}^{(0)} \cos \left(2 \psi_{1}\right)+\alpha_{2}^{(2)} \alpha_{1}^{(0)} \cos \left(2 \psi_{2}\right)-\alpha_{1}^{(2)} \alpha_{2}^{(2)} \cos \left(2 \psi_{1}+2 \psi_{2}\right)\right)
$$

Strong tension/strong anisotropy.

$$
\begin{aligned}
\frac{E}{\pi}= & \frac{2 \alpha_{1}^{(0)} \alpha_{2}^{(0)}}{\mu^{2}} K_{0}(R / \mu)+\frac{\alpha_{1}^{(2)} \alpha_{2}^{(2)}}{\mu^{2}} \cos \left(2\left(\psi_{1}-\psi_{2}\right)\right) K_{0}(R / \mu) \\
& +\frac{2 K_{2}(R / \mu)}{\mu^{2}}\left(\alpha_{1}^{(2)} \alpha_{2}^{(0)} \cos 2 \psi_{1}+\alpha_{1}^{(0)} \alpha_{2}^{(2)} \cos 2 \psi_{2}\right) \\
& +\frac{\alpha_{1}^{(2)} \alpha_{2}^{(2)}}{\mu^{2}} \cos \left(2\left(\psi_{1}+\psi_{2}\right)\right)\left(-\frac{48 \mu^{4}}{R^{4}}+K_{4}(R / \mu)\right) .
\end{aligned}
$$

4.1. Comparison with other results. First we note that when there is no anisotropy and no tension (4.1) reduces to the energy depending on the fourth power of the inverse separation previously derived [17, 40, 13, 26], describing the repulsion of two isotropic inclusions.

We first compare our results to previous works which consider anisotropic inclusions with no tension. Formula (2.4) in [26] gives the interaction energy as

$$
E=-\frac{A^{2} \kappa d_{1}^{2} Q_{2}^{2}}{16 \pi R^{2}} \cos \left(2 \theta_{1}+2 \theta_{2}\right),
$$

which, in our notation, after nondimensionalisation is

$$
E=-\frac{4 \pi}{R^{2}}\left(\frac{\varepsilon^{4} L^{2} d_{1}^{2} Q_{2}^{2}}{64 \xi^{2}}\right) \cos \left(2 \psi_{1}+2 \psi_{2}\right) .
$$

If we choose

$$
\alpha_{1}^{(2)}=\alpha_{2}^{(2)}=\frac{d_{1} Q_{2} L \varepsilon^{2}}{8 \xi}, \quad \alpha_{1}^{(0)}=\alpha_{2}^{(0)}=0,
$$

then this agrees with our no tension-strong anisotropy limit in which there is no isotropic contribution, corresponding to the anisotropy parameters $\omega_{1}=\omega_{2}=\infty^{1}$.

\footnotetext{
${ }^{1}$ Note that since $x$ and $y$ were scaled with $L$, and $h$ was scaled with $\xi L$, the unscaled opening angle forced by the inclusion is $\xi \alpha / \varepsilon$.
} 
Similarly, formula (1) of [25] is (in their notation, see also formula (10) of [10])

$$
E=\frac{16 \pi r_{\text {rod }}^{4}}{9 R^{2}} \kappa C_{r 1} C_{r 2}\left(\cos \left(2 \theta_{1}\right)+\cos \left(2 \theta_{2}\right)-\cos \left(2 \theta_{1}-2 \theta_{2}\right)\right) .
$$

In our notation $\left(\psi_{1}=\theta_{1}, \psi_{2}=\pi-\theta_{2}\right)$, after nondimensionalisation, this is

$$
E=\frac{4 \pi}{R^{2}}\left(\frac{4 \varepsilon^{4} L^{2} C_{r 1} C_{r 2}}{9 \xi^{2}}\right)\left(\cos \left(2 \psi_{1}\right)+\cos \left(2 \psi_{2}\right)-\cos \left(2 \psi_{1}+2 \psi_{2}\right)\right) .
$$

This corresponds to our no tension-strong anisotropy result if we set

$$
\alpha_{1}^{(0)}=\alpha_{1}^{(2)}=\frac{2 \varepsilon^{2} L C_{r 1}}{3 \xi}, \quad \alpha_{2}^{(0)}=\alpha_{2}^{(2)}=\frac{2 \varepsilon^{2} L C_{r 2}}{3 \xi} .
$$

Thus their approach fixes the isotropic and anisotropic components to be the same size, so that the anisotropy parameters $\omega_{1}=\omega_{2}=1$.

Finally we compare our formulae to previous results involving tension. First we observe that our strong tension-weak anisotropy limit agrees with formula (23) of [40] noting that their $\xi=1 / \mu L$ and their $\alpha_{i}=\xi \alpha_{0}^{(0)} / \varepsilon$ in our notation.

There are two nonzero tension results presented in formulae (5) and (6) of [25] in the limit of inclusion separation $R \gg \xi$ (their notation), where $\xi$ is the lengthscale over which bending and tension effects are comparable. Their $\xi$ is $\mu L$ in our notation, and the corresponding limit is $R \gg \mu$ (our dimensionless $R$ ). The first result supposes the inclusion orientations satisfy $\cos \left(2 \theta_{1}-2 \theta_{2}\right) \neq 0$, and is (their notation)

$$
E=-\frac{64 \pi r_{\text {rod }}^{4} \xi^{2}}{3 R^{4}} \kappa C_{r 1} C_{r 2} \cos \left(2 \theta_{1}-2 \theta_{2}\right) .
$$

In our notation, after nondimensionalisation, this is

$$
E=-\frac{64 \pi \varepsilon^{4} \mu^{2} L^{2} C_{r 1} C_{r 2}}{3 R^{4} \xi^{2}} \cos \left(2 \psi_{1}+2 \psi_{2}\right) .
$$

Since $K_{n}(r) \sim \sqrt{(\pi / 2 r)} \mathrm{e}^{-r}$ as $r \rightarrow \infty$, when $R \gg \mu$ (4.4) becomes

$$
E \sim-\frac{48 \pi \mu^{2} \alpha_{1}^{(2)} \alpha_{2}^{(2)}}{R^{4}} \cos \left(2 \psi_{1}+2 \psi_{2}\right) .
$$

The results agree if

$$
\alpha_{1}^{(2)}=\frac{2 \varepsilon^{2} L C_{r 1}}{3 \xi}, \quad \alpha_{2}^{(2)}=\frac{2 \varepsilon^{2} L C_{r 2}}{3 \xi},
$$

which is consistent with (4.5). The second result supposes the inclusion orientations satisfy $\cos \left(2 \theta_{1}-2 \theta_{2}\right)=0$, and is (their notation)

$$
E=\frac{2 \sqrt{2} \pi^{3 / 2} r_{\mathrm{rod}}^{4}}{9 \xi^{3 / 2}} \frac{e^{-R / \xi}}{\sqrt{R}} \kappa C_{r 1} C_{r 2}\left(2+2 \cos 2 \theta_{1}+2 \cos 2 \theta_{2}+\cos \left(2 \theta_{1}+2 \theta_{2}\right)\right)
$$

In our notation, after nondimensionalisation, this is (4.10)

$$
E=\frac{2 \sqrt{2} \pi^{3 / 2} \varepsilon^{4} L^{2}}{9 \mu^{3 / 2} \xi^{2}} \frac{e^{-R / \mu}}{\sqrt{R}} C_{r 1} C_{r 2}\left(2+2 \cos 2 \psi_{1}+2 \cos 2 \psi_{2}+\cos \left(2 \psi_{1}-2 \psi_{2}\right)\right) .
$$


With $\cos \left(2 \psi_{1}+2 \psi_{2}\right)=0$, when $R \gg \mu,(4.4)$ becomes

$$
\begin{aligned}
E \sim \frac{\pi^{3 / 2} \mathrm{e}^{-R / \mu}}{(2 R)^{1 / 2} \mu^{3 / 2}}( & 2 \alpha_{1}^{(0)} \alpha_{2}^{(0)}+\alpha_{1}^{(2)} \alpha_{2}^{(2)} \cos \left(2 \psi_{1}-2 \psi_{2}\right) \\
& \left.+2 \alpha_{1}^{(2)} \alpha_{2}^{(0)} \cos 2 \psi_{1}+2 \alpha_{1}^{(0)} \alpha_{2}^{(2)} \cos 2 \psi_{2}\right),
\end{aligned}
$$

which agrees with (4.10) if we impose (4.5).

\section{Remarks on the interaction energies.}

5.1. Weak anisotropy/weak tension. We consider for illustration purpose the case of two identical inclusions, so that $\alpha_{1}^{(0)}=\alpha_{2}^{(0)}$ and $\bar{\alpha}_{1}^{(2)}=\bar{\alpha}_{2}^{(2)}=\bar{\omega} \alpha_{1}^{(0)}$. We write $R=\lambda^{1 / 2} \hat{R}, \bar{\omega}=\hat{\omega} / \lambda, E=4 \pi \varepsilon^{2} \alpha_{1}^{(0)} \alpha_{2}^{(0)} \lambda^{-2} \bar{E}$, to give the non-constant part of the interaction energy as

$$
\bar{E}=\frac{\hat{\omega}}{\hat{R}^{2}}\left(\cos 2 \psi_{1}+\cos 2 \psi_{2}\right)+\frac{2}{\hat{R}^{4}}-\frac{\log \hat{R}}{2} .
$$

We calculate the (rescaled) torque $\tau_{i}$ exerted on the $i$ th inclusion around the $z$-axis as a result of the membrane-mediated influence induced by the other inclusion by taking the negative partial derivative of (5.1) with respect to $\psi_{i}$ :

$$
\tau_{i}=\frac{2 \hat{\omega} \sin 2 \psi_{i}}{\hat{R}^{2}}
$$

We see that the inclusions are in rotational equilibrium when $\psi_{i}=n \pi / 2$ for $n \in \mathbb{Z}$. Each inclusion has two possible equilibrium configurations: a high energy state in which $\psi_{i}=0$ (or $\pi$ ) and the low energy state in which $\psi_{i}=\pi / 2$ (or $3 \pi / 2$ ).

We calculate the (rescaled) effective force $F$ between the inclusions by taking the negative partial derivative of (5.1) with respect to $R$, to give

$$
F=\frac{2 \hat{\omega}}{\hat{R}^{3}}\left(\cos 2 \psi_{1}+\cos 2 \psi_{2}\right)+\frac{8}{\hat{R}^{5}}+\frac{1}{2 \hat{R}} .
$$

The first term may be attractive or repulsive depending on the orientations of the two inclusions. In particular we note that two inclusions in the high energy state repel at large distances, while two inclusions in the low energy state attract at large distances. For anisotropic inclusions, attraction will occur when their semi-major axes are aligned in an "end-to-end" configuration. This phenomenon has also been observed in large-scale molecular dynamics simulations [33].

Assuming the inclusions are in rotational equilibrium the force is

$$
F=\frac{1}{2 \hat{R}}-\frac{4 \hat{\omega}}{\hat{R}^{3}}+\frac{8}{\hat{R}^{5}} .
$$

At both small and large distances the force is repulsive.

However, for $\hat{\omega}>1$ (i.e. provided the tension is sufficiently weak or the anisotropy sufficiently strong) there is a bifurcation in which a stable and an unstable equilibrium point appear. These equilibrium separations are shown as a function of $\hat{\omega}$ in Fig. 5 .

5.2. Strong tension/strong anisotropy. Again consider the case $\alpha_{1}^{(0)}=\alpha_{2}^{(0)}$, $\alpha_{1}^{(2)}=\alpha_{2}^{(2)}=\omega \alpha_{1}^{(0)}$. We write $R=\bar{R} \mu, E=\pi \alpha_{1}^{(0)} \alpha_{2}^{(0)} / \mu^{2} \bar{E}$ to give 


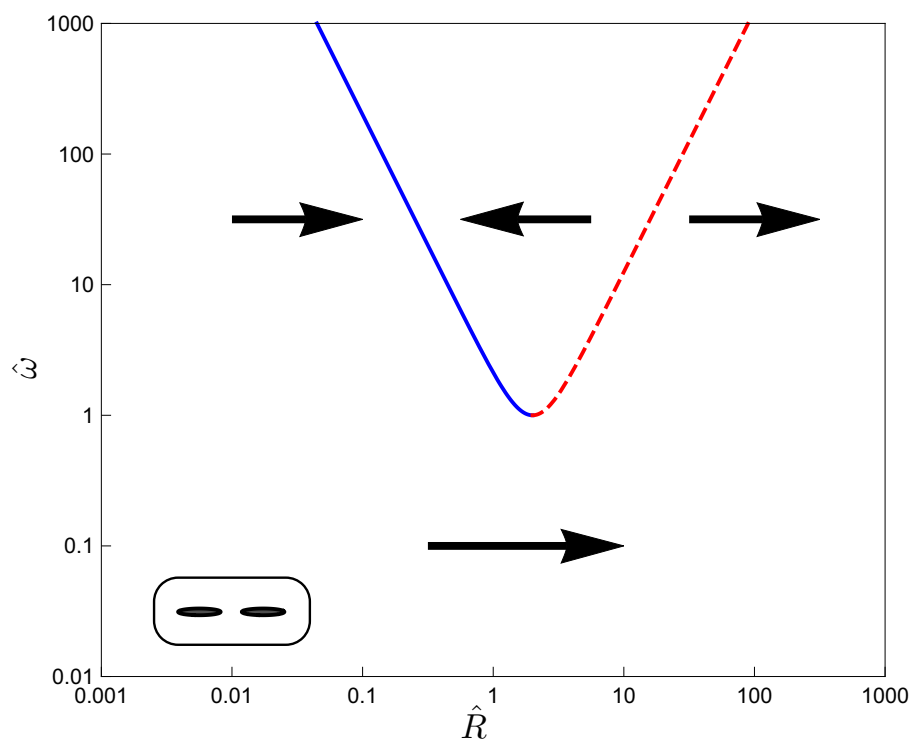

FIG. 5. Equilibrium positions in the $(\hat{R}, \hat{\omega})$-plane. The dashed red line is an unstable equilibrium separation and the solid blue line is a stable equilibrium separation, which exist for $\hat{\omega}>1$. The arrows indicate the direction of the force between the inclusions. The inset shows the stable orientation of the inclusions.

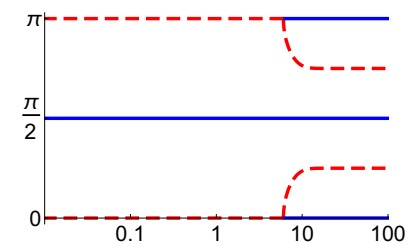

(a) $\omega=0.05$

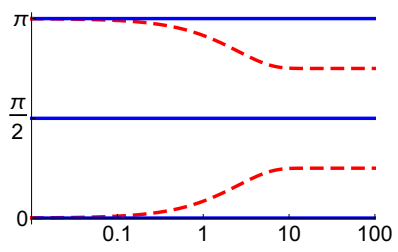

(b) $\omega=0.5$

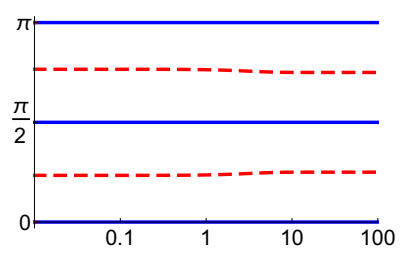

(c) $\omega=5$

FIG. 6. Equilibrium orientations as a function of $\bar{R}$ for various values of $\omega$ for the solution in which $\psi_{1}=\psi_{2}$. Solid blue lines are stable, dashed red lines unstable (within the class of solutions in which $\psi_{1}=\psi_{2}$ ).

$$
\begin{aligned}
\bar{E}=2 & K_{0}(\bar{R})+2 \omega K_{2}(\bar{R})\left(\cos 2 \psi_{1}+\cos 2 \psi_{2}\right) \\
& +\omega^{2} \cos \left(2\left(\psi_{1}-\psi_{2}\right)\right) K_{0}(\bar{R})+\omega^{2} \cos \left(2\left(\psi_{1}+\psi_{2}\right)\right)\left(K_{4}(\bar{R})-\frac{48}{\bar{R}^{4}}\right) .
\end{aligned}
$$

There are zero-torque solutions in which $\psi_{1}=\psi_{2}$ and zero-torque solutions in which $\psi_{1}=-\psi_{2}$. In Fig. 6 we show the bifurcation diagram for zero-torque solutions in which $\psi_{1}=\psi_{2}$ as a function of $\bar{R}$ for various values of $\omega$. Stability is indicated within the class $\psi_{1}=\psi_{2}$. In Fig. 7 we show the bifurcation diagram for zerotorque solutions in which $\psi_{1}=-\psi_{2}$ as a function of $\bar{R}$ for various values of $\omega$. Stability is indicated within the class $\psi_{1}=-\psi_{2}$. Noting that the solutions in which $\psi_{1}=\psi_{2}=\pi / 2$ are equivalent to those in which $\psi_{1}=-\psi_{2}=\pi / 2$, we see that the only stable solutions are those shown in Fig. 7. Perhaps surprisingly, we see that for sufficiently strong anisotropy and sufficiently strong tension (or sufficiently large separation) the stable configuration for $\psi_{1}$ and $\psi_{2}$ is neither 0 nor $\pi / 2$ but some 


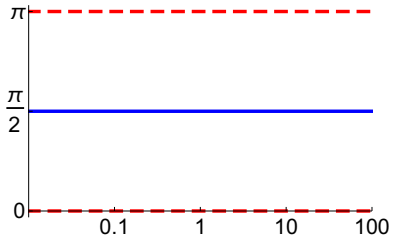

(a) $\omega=1$

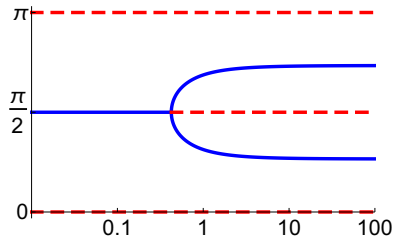

(b) $\omega=10$

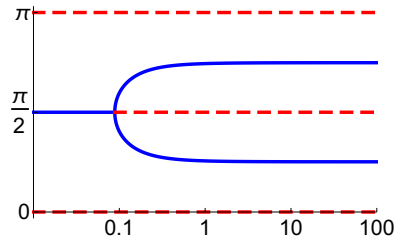

(c) $\omega=100$

FIG. 7. Equilibrium orientations as a function of $\bar{R}$ for various values of $\omega$ for the solution in which $\psi_{1}=-\psi_{2}$. Solid blue lines are stable, dashed red lines unstable (within the class of solutions in which $\left.\psi_{1}=-\psi_{2}\right)$.

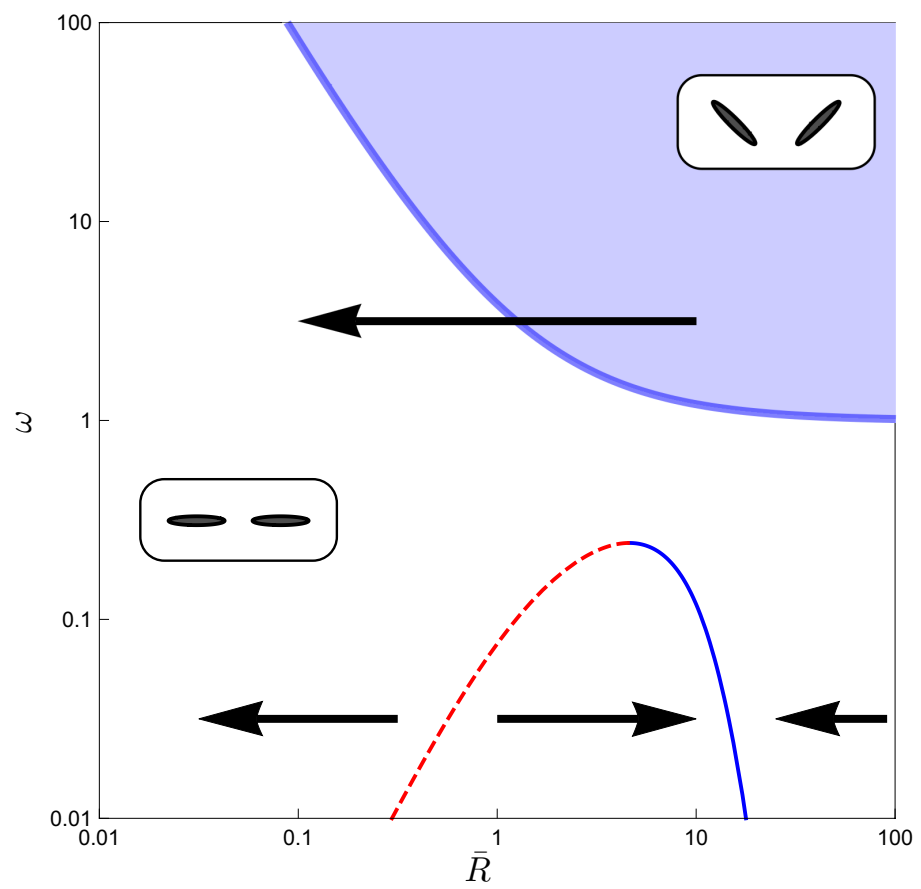

FIG. 8. Equilibrium orientations and positions in the $(\bar{R}, \omega)$-plane. In the white region the stable zero-torque solution is $\psi_{1}=\psi_{2}=\pi / 2$. There is a pitchfork bifurcation at the boundary of this region, and in the blue region the stable zero-torque solutions are $\psi_{1}=\pi / 2 \pm \delta \psi, \psi_{2}=\pi / 2 \mp \delta \psi$, with $\delta \psi$ varying from zero at the bifurcation to $\pi / 4$ at infinity. The dashed red line is an unstable equilibrium separation and the solid blue line is a stable equilibrium separation, which exist for $\omega<\omega_{c} \approx 0.242$. The arrows indicate the direction of the force between the inclusions.

intermediate angle, which approaches $\pi / 4$ as $R \rightarrow \infty$, a regime that has not been observed before. The bifurcation in Fig. 7 exists only for $\omega>1$, so that it is not present in the results [25], whose formulae correspond to the case $\omega=1$.

In Fig. 8 we show the stable orientations as a function of $\bar{R}$ and $\omega$. Also shown are the equilibrium separations. For $\omega>\omega_{c} \approx 0.242$ the inclusions attract at all distances. For $\omega<\omega_{c}$ there is a bifurcation in which stable and unstable equilibrium separations appear.

By comparing Figs. 8 and 5 we see that they are consistent: the bottom left corner of Fig. 8 matches with the top right corner of Fig. 5. Together they predict a region of bistability. For $\omega>\omega_{c}$ inclusions are attracted to the stable equilibrium point in Fig. 
5 (in which the separation is small). For For $\omega<\omega_{c}$ and $\hat{\omega}=\omega \mu / \varepsilon=\omega \sqrt{\kappa / T r_{0}^{2}}<1$ they are attracted to the stable equilibrium point in Fig. 8 (in which the separation is large). But for $r_{0} \sqrt{T / \kappa}<\omega<\omega_{c}$ both equilibria exist and are stable. By forming a uniform composite expansion between the equilibrium position $\omega(\bar{R})$, shown in Fig. 8, and $\hat{\omega}(\hat{R})$ shown in Fig. 5 we obtain the equilibrium separation over the whole range of $R$, as shown in Fig. 1 .

6. Conclusion. Using matched asymptotic expansions we have examined the membrane-mediated interactions between symmetry-breaking inclusions embedded on an approximately flat fluid membrane. For simplicity we considered circular inclusions and broke symmetry by imposing a spatially dependent contact angle. A similar analysis can be performed for non-circular inclusions. In the case of weak anisotropy, imposing a constant contact angle on the perturbed boundary

$$
\rho_{i}\left(\phi_{i}\right)=1-\varepsilon^{2} \omega_{i} \cos \left(2 \phi_{i}-2 \psi_{i}\right),
$$

turns out to give the same interaction energy to leading order(although the details of the asymptotic expansions are different). In the strong anisotropy regime it is algebraically more complicated to analyze geometric symmetry breaking, but our general approach is still applicable and we expect the results to be similar.

In the absence of membrane tension we find the previously known isotropic repulsion with energy proportional to the inverse separation to the fourth power, as well as an additional orientation-dependent interaction proportional to the inverse separation squared. The dominant symmetry-breaking effect is the quadrupole term, invariant under a rotation of an individual inclusion by $\pi$.

When we include membrane tension we find that the interaction is attractive at very large distances for any non-zero anisotropy $\omega$, so that there is always at least one stable equilibrium separation. In general, we find an $S$-shaped dependence of the equilibrium separation on anisotropy, with bi-stability for $r_{0} \sqrt{T / \kappa}<\omega<0.242$.

We find that our interaction energy is a generalization of that presented in [25], in which inclusions are modeled as point singularities (in effect they focus solely in the regime we term the outer region) with the resulting infinities later regularized using a cut-off in Fourier space. In particular their formulae correspond to the case $\omega=1$, so that they do not capture either the pitchfork bifurcation in equilibrium orientation and the fold bifurcation in equilibrium position illustrated in Fig. 8.

Although our analysis has been performed for just two inclusions, it is clear how to generalize the interaction to the general case of $N$ inclusions. In both parameter regimes we have considered, the outer solution will be a sum of logarithms centered at each inclusion. For the strong anisotropy/strong tension case, the second-order inner solution will now have a sum of terms matching with each inclusion, and since the relevant term in the energy arises from integrating $h_{\ell}^{(0)}, h_{\ell}^{(2)}$ the energy of the configuration will simply be the sum of the pairwise interaction energies. However, in the weak anisotropy/weak tension case, the calculation of the energy is more delicate, and there will be multiple cross-terms arising from integrating the quadratic membrane energy (2.2) over the outer region. In that case the configuration energy is not simply the sum of the pairwise interaction energies. In the many-inclusion case a natural and fundamental question to pursue is the nature and stability of equilibrium configurations, and the mechanisms by which large numbers of inclusions will dynamically self-assemble. A full understanding of this self-assembly process is crucial for many cell-shaping phenomena. 


\section{REFERENCES}

[1] H. Agrawal, L. Liu, and P. Sharma, Revisiting the curvature-mediated interactions between proteins in biological membranes, Soft matter, 12 (2016), pp. 8907-8918.

[2] H. Aranda-Espinoza, A. Berman, N. Dan, P. Pincus, and S. Safran, Interaction between inclusions embedded in membranes, Biophysical journal, 71 (1996), pp. 648-656.

[3] T. F. BAnchoff And S. T. Lovet, Differential geometry of curves and surfaces, CRC Press, 2016.

[4] M. F. Brown, Soft matter in lipid-protein interactions, Annual review of biophysics, 46 (2017), pp. 379-410.

[5] B. R. Capraro, Y. Yoon, W. Cho, and T. Baumgart, Curvature sensing by the Epsin NTerminal Homology domain measured on cylindrical lipid membrane tethers, Journal of the American Chemical Society, 132 (2010), pp. 1200-1201, https://doi.org/10.1021/ja907936c, http://dx.doi.org/10.1021/ja907936c.

[6] N. Dan, P. Pincus, And S. SAFran, Membrane-induced interactions between inclusions, Langmuir, 9 (1993), pp. 2768-2771.

[7] L. Danne, M. Aktas, A. Unger, W. A. Linke, R. Erdmann, and F. Narberhaus, Membrane remodeling by a bacterial phospholipid-methylating enzyme, mBio, 8 (2017), pp. e02082-16.

[8] D. S. Dean, V. A. PARsegian, And R. PodgornikA, Fluctuation mediated interactions due to rigidity mismatch and their effect on miscibility of lipid mixtures in multicomponent membranes, Journal of Physics: Condensed Matter, 27 (2015), p. 214004

[9] P. Dommersnes And J.-B. Fournier, Casimir and mean-field interactions between membrane inclusions subject to external torques, EPL (Europhysics Letters), 46 (1999), p. 256.

[10] P. Dommersnes And J.-B. Fournier, N-body study of anisotropic membrane inclusions: Membrane mediated interactions and ordered aggregation, The European Physical Journal BCondensed Matter and Complex Systems, 12 (1999), pp. 9-12.

[11] P. G. Dommersnes AND J.-B. Fournier, The many-body problem for anisotropic membrane inclusions and the self-assembly of "saddle" defects into an "egg carton", Biophysical journal, 83 (2002), pp. 2898-2905.

[12] A. Evans, M. Turner, And P. SEns, Interactions between proteins bound to biomembranes, Physical Review E, 67 (2003), p. 041907.

[13] J. Fournier And P. Dommersnes, Comment on" long-range forces in heterogeneous fluid membranes", EPL (Europhysics Letters), 39 (1997), p. 681.

[14] J.-B. Fournier AND P. GAlatola, High-order power series expansion of the elastic interaction between conical membrane inclusions, The European Physical Journal E, 38 (2015), p. 86.

[15] R. Golestanian, M. Goulian, and M. Kardar, Fluctuation-induced interactions between rods on a membrane, Physical Review E, 54 (1996), p. 6725.

[16] R. Golestanian, M. Goulian, And M. KARDAR, Fluctuation-induced interactions between rods on membranes and interfaces, EPL (Europhysics Letters), 33 (1996), p. 241.

[17] M. Goulian, R. Bruinsma, And P. Pincus, Long-range forces in heterogeneous fluid membranes, EPL (Europhysics Letters), 22 (1993), p. 145.

[18] W. Helfrich, Elastic properties of lipid bilayers: Theory and possible experiments, Zeitschrift für Naturforschung, C: Journal of Biosciences, 28 (1973), pp. 693-703, https://doi.org/10. 1515/znc-1973-11-1209.

[19] J. D. JACKSON, Classical electrodynamics, AAPT, 1999.

[20] L. Johannes, W. Pezeshikinn, J. H. Ipsen, And J. C. Shillcock, Clustering on membranes: Fluctuations and more, Trends in Cell Biology, (2018).

[21] G. KOLESKI AND J.-B. FOURNIER, Linear response approximation in effective field theory for the calculation of elastically mediated interactions in one dimension, Physical Review E, 93 (2016), p. 052128.

[22] J. Kwiecinski, S. J. Chapman, And A. Goriely, Self-assembly of a flament by curvatureinducing proteins, Physica D: Nonlinear Phenomena, 344 (2017), pp. 68-80.

[23] H.-K. Lin, R. Zandi, U. Mohideen, And L. P. Pryadko, Fluctuation-induced forces between inclusions in a fluid membrane under tension, Physical review letters, 107 (2011), p. 228104.

[24] H. T. MCMAhon And J. L. Gallop, Membrane curvature and mechanisms of dynamic cell membrane remodelling, Nature, 438 (2005), p. 590.

[25] H. Noguchi And J.-B. Fournier, Membrane structure formation induced by two types of banana-shaped proteins, Soft matter, 13 (2017), pp. 4099-4111.

[26] J.-M. PARK AND T. LUBENSKY, Interactions between membrane inclusions on fluctuating membranes, Journal de Physique I, 6 (1996), pp. 1217-1235.

[27] B. J. Peter, H. M. Kent, I. G. Mills, Y. Vallis, P. J. G. Butler, P. R. Evans, And 
H. T. McMahon, BAR domains as sensors of membrane curvature: The amphiphysin BAR structure, Science, 303 (2004), pp. 495-499, https://doi.org/10.1126/science.1092586, http://science.sciencemag.org/content/303/5657/495.abstract.

[28] B. J. Reynwar and M. Deserno, Membrane-mediated interactions between circular particles in the strongly curved regime, Soft Matter, 7 (2011), pp. 8567-8575.

[29] B. J. Reynwar, G. Illya, V. A. Harmandaris, M. M. Müller, K. Kremer, and M. DeSERNO, Aggregation and vesiculation of membrane proteins by curvature-mediated interactions, Nature, 447 (2007), pp. 461-464.

[30] K. SaPp AND L. Maibaum, Suppressing membrane height fluctuations leads to a membranemediated interaction among proteins, Physical Review E, 94 (2016), p. 052414.

[31] M. Simunovic AND P. BAssereau, Reshaping biological membranes in endocytosis: crossing the configurational space of membrane-protein interactions, Biological Chemistry., 395 (2014), pp. 275-283, https://doi.org/10.1515/hsz-2013-0242.

[32] M. Simunovic, C. Mim, T. C. Marlovits, G. Resch, V. M. Unger, and G. A. Voth, Protein-mediated transformation of lipid vesicles into tubular networks, Biophysical Journal, 105 (2013), pp. 711-719, https://doi.org/10.1016/j.bpj.2013.06.039, http://dx.doi.org/ $10.1016 /$ j.bpj.2013.06.039.

[33] M. Simunovic, A. Šarić, J. M. Henderson, K. Y. C. Lee, and G. A. Voth, Long-range organization of membrane-curving proteins, ACS central science, 3 (2017), pp. 1246-1253.

[34] M. Simunovic, A. SRivastava, And G. A. Voth, Linear aggregation of proteins on the membrane as a prelude to membrane remodeling, Proceedings of the National Academy of Sciences, 110 (2013), pp. 20396-20401, https://doi.org/10.1073/pnas.1309819110, http://www.pnas.org/content/110/51/20396.abstract.

[35] M. Simunovic And G. A. Voth, Membrane tension controls the assembly of curvaturegenerating proteins, Nature Communications, 6 (2015), p. 7219, http://dx.doi.org/10.1038/ ncomms8219.

[36] M. Simunovic, G. A. Voth, A. Callan-Jones, and P. Bassereau, When physics takes over: Bar proteins and membrane curvature, Trends in cell biology, 25 (2015), pp. 780-792.

[37] B. Sorre, A. Callan-Jones, J. Manzi, B. Goud, J. Prost, P. Bassereau, and A. Roux, Nature of curvature coupling of amphiphysin with membranes depends on its bound density, Proceedings of the National academy of Sciences of the United States of America, 109 (2012), pp. 173-178, https://doi.org/10.1073/pnas.1103594108.

[38] A. Suarez, T. Ueno, R. Huebner, J. M. McCaffery, and T. Inoue, Bin/amphiphysin/rvs (bar) family members bend membranes in cells, Scientific reports, 4 (2014), p. 4693.

[39] C. Van Der Wel, A. Vahid, A. Šarić, T. Idema, D. Heinrich, and D. J. Kraft, Lipid membrane-mediated attraction between curvature inducing objects, Scientific reports, 6 (2016), p. 32825

[40] T. Weikl, M. Kozlov, And W. Helfrich, Interaction of conical membrane inclusions: Effect of lateral tension, Physical Review E, 57 (1998), p. 6988.

[41] C. Yolcu And M. Deserno, Membrane-mediated interactions between rigid inclusions: an effective field theory, Physical Review E, 86 (2012), p. 031906.

[42] C. Yoldu, R. C. Haussman, and M. Deserno, The effective field theory approach towards membrane-mediated interactions between particles, Advances in colloid and interface science, 208 (2014), pp. 89-109. 